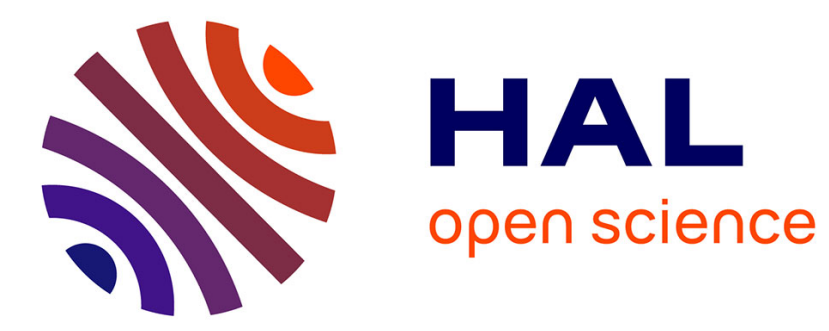

\title{
Strain localization analysis for single crystals and polycrystals: Towards microstructure-ductility linkage
}

Gérald Franz, Farid Abed-Meraim, Marcel Berveiller

\section{To cite this version:}

Gérald Franz, Farid Abed-Meraim, Marcel Berveiller. Strain localization analysis for single crystals and polycrystals: Towards microstructure-ductility linkage. International Journal of Plasticity, 2013, 48, pp.1-33. 10.1016/j.ijplas.2013.02.001 . hal-01081928

\section{HAL Id: hal-01081928 \\ https://hal.science/hal-01081928}

Submitted on 12 Nov 2014

HAL is a multi-disciplinary open access archive for the deposit and dissemination of scientific research documents, whether they are published or not. The documents may come from teaching and research institutions in France or abroad, or from public or private research centers.
L'archive ouverte pluridisciplinaire HAL, est destinée au dépôt et à la diffusion de documents scientifiques de niveau recherche, publiés ou non, émanant des établissements d'enseignement et de recherche français ou étrangers, des laboratoires publics ou privés. 


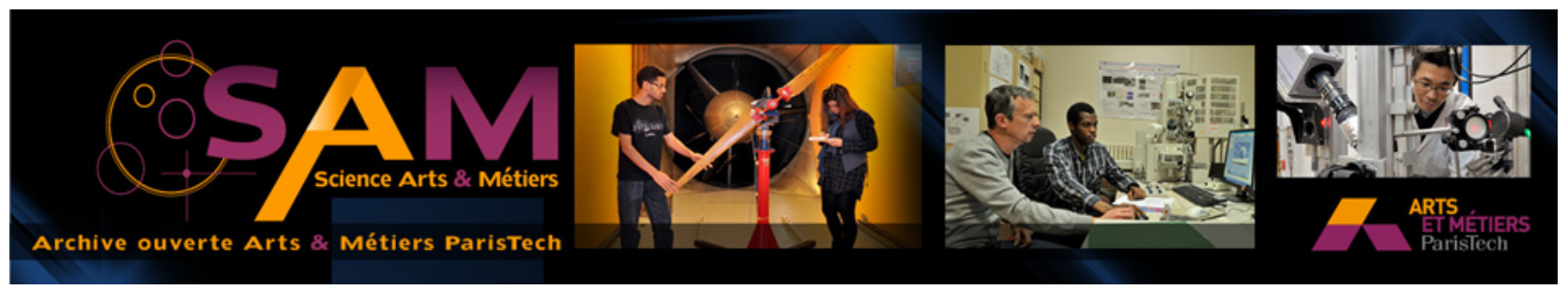

Science Arts \& Métiers (SAM)

is an open access repository that collects the work of Arts et Métiers ParisTech researchers and makes it freely available over the web where possible.

This is an author-deposited version published in: http://sam.ensam.eu

Handle ID: .http://hdl.handle.net/10985/8875

\section{To cite this version :}

Gérald FRANZ, Farid ABED-MERAIM, Marcel BERVEILLER - Strain localization analysis for single crystals and polycrystals: Towards microstructure-ductility linkage - International Journal of Plasticity - Vol. 48, p.1-33 - 2013 


\section{Accepted Manuscript}

Strain localization analysis for single crystals and polycrystals: towards microstructure-ductility linkage

Gérald Franz, Farid Abed-Meraim, Marcel Berveiller

PII:

S0749-6419(13)00030-2

DOI: http://dx.doi.org/10.1016/j.ijplas.2013.02.001

Reference: INTPLA 1619

To appear in:

International Journal of Plasticity

Received Date: $\quad 13$ May 2012

Revised Date: $\quad 24$ January 2013

Please cite this article as: Franz, G., Abed-Meraim, F., Berveiller, M., Strain localization analysis for single crystals and polycrystals: towards microstructure-ductility linkage, International Journal of Plasticity (2013), doi: http:// dx.doi.org/10.1016/j.ijplas.2013.02.001

This is a PDF file of an unedited manuscript that has been accepted for publication. As a service to our customers we are providing this early version of the manuscript. The manuscript will undergo copyediting, typesetting, and review of the resulting proof before it is published in its final form. Please note that during the production process errors may be discovered which could affect the content, and all legal disclaimers that apply to the journal pertain. 


\title{
Strain localization analysis for single crystals and polycrystals: towards microstructure-ductility linkage
}

Gérald FRANZ ${ }^{\mathrm{a}}$, Farid ABED-MERAIM ${ }^{\mathrm{b}, \mathrm{c}, *}$, Marcel BERVEILLER ${ }^{\mathrm{b}}$

${ }^{\text {a }}$ LTI, EA 3899 - IUT Amiens, Avenue des Facultés - Le Bailly, 80025 Amiens Cedex 1, France

${ }^{\mathrm{b}}$ LEM3, UMR CNRS 7239 - Arts et Métiers ParisTech, 4 rue Augustin Fresnel, 57078 Metz Cedex 3, France

${ }^{\mathrm{c}}$ DAMAS, Laboratory of Excellence on Design of Alloy Metals for low-mAss Structures, Université de Lorraine, France

\begin{abstract}
In this paper, we performed a strain localization analysis for single crystals and polycrystals, with the specific aim of establishing a link between the microstructure-related parameters and ductility. To this end, advanced large-strain elastic-plastic single crystal constitutive modeling is adopted, accounting for the key physical mechanisms that are relevant at the microscale, such as dislocation storage and annihilation. The self-consistent scale-transition scheme is then used to derive the overall constitutive response of polycrystalline aggregates, including the essential microstructural aspects (e.g., initial and induced textures, dislocation density evolution, and softening mechanisms). The resulting constitutive equations for single crystals and polycrystals are coupled with two strain localization criteria: bifurcation theory, which is also related to the loss of ellipticity in the associated boundary value problem, and the strong ellipticity condition, which is presented in full detail along with mathematical links allowing for hierarchical classification in terms of conservativeness. The application of the proposed coupling to single crystals and polycrystals allows the effect of physical microstructural parameters on material ductility to be investigated. Consistent results are found for both single crystals and polycrystals. In addition, forming limit diagrams (FLDs) are constructed for IF-Ti single-phase steels with comparison to the reference results, demonstrating the predictive capability of the proposed approach in investigations of sheet metal formability. The results of the self-consistent scheme are systematically compared to those of the more classical full-constraint Taylor model, both in terms of the impact of microstructural parameters on ductility and in terms of the predicted formability limits and the level of the associated limit strains. Finally, we investigated the impact of strain-path changes on formability through the analysis of the effect of prestrain on the FLDs.
\end{abstract}

Keywords: Plastic instabilities; Rice's bifurcation criterion; Loss of strong ellipticity; Crystal plasticity; Self-consistent scale transition; Microstructure-ductility relationships

* Corresponding author. Tel.: +(33) 3.87.37.54.79; fax: +(33) 3.87.37.54.70.

E-mail address: farid.abed-meraim@ensam.eu (F. Abed-Meraim). 


\section{Introduction}

In the literature dealing with plastic instabilities in general and more recent studies on the prediction of plastic instabilities in the sheet metal forming process, many instability criteria have been developed, and some of these criteria have been extensively applied to sheet metals to investigate their formability limits. Exhaustively reviewing these criteria is difficult, considering the multitude of variants deriving from some of these approaches. However, a review of the literature reveals that the criteria can be classified into at least four distinct categories depending on their fundamental basis and theoretical or physical background.

For stretched sheet metals, two forms of necking, namely diffuse and localized necking, may occur. It has been shown that diffuse necking occurs prior to localized necking, and it is now well recognized that the maximum allowable straining in sheet metal forming is determined by localized necking. For this reason, forming limit diagrams (FLDs) are commonly determined at localization in most of the current formability approaches, and from an experimental perspective, the FLDs are obtained at localized necking for various loading paths (e.g., uniaxial tensile test, plane strain tension, biaxial tensile tests, etc.) by means of Marciniak's or Nakazima's punch with specimens of different widths.

Early instability criteria were based on the maximum force principle (Considère, 1885), and its two-dimensional extension (Swift, 1952) was used for sheet metal applications. In their original form, these criteria were intended to allow for the prediction of diffuse necking. Later, these maximum-force-based criteria were extended to the prediction of localized necking, and some enhanced versions were developed to take certain effects, such as those related to thickness and the strain path, into account (Hora et al., 1996; Mattiason et al., 2006). Note also that Hill's zeroextension criterion (Hill, 1952), which predicts localized necking on the left-hand side of the FLD, was developed during the same time as Swift's diffuse necking criterion.

Another approach, which postulates a pre-existing defect in the material sheet, was proposed by Marciniak and Kuczynski (Marciniak and Kuczynski, 1967). In its original form, the M-K model can be regarded as a complementary approach to Hill's zero-extension criterion, which is only applicable to the left-hand side of the FLD, as no zero-extension direction exists for positive biaxial stretching. However, because localized necking in biaxial stretching is observed in practice, a preexisting defect has to be introduced in the $\mathrm{M}-\mathrm{K}$ model to capture this phenomenon, which may provide some justification for this imperfection theory. Subsequently, the M-K criterion has been extended to the left-hand side of the FLD by allowing the pre-existing geometrical defect, which is 
represented by an oriented band of reduced thickness, to rotate in the sheet plane (Hutchinson and Neale, 1978).

In addition to the aforementioned engineering approaches, another category of plastic instability criteria was developed based on a more fundamental background. Drucker and Hill's theory (Drucker, 1950; Drucker, 1956; Hill, 1958), also referred to as the general bifurcation criterion, represents another class of approaches for necking prediction. This condition of positiveness of the second-order work provides a lower bound for all of the bifurcation-based criteria in this category. In the same class of criteria, Valanis (1989) suggested using a limit-point bifurcation criterion, which is less conservative than the general bifurcation criterion but coincides with it within the framework of associative plasticity and small strains. Note that a more conservative criterion will predict earlier plastic instability associated with lower critical strains. With regard to localized modes of deformation, Rudnicki and Rice (1975), Stören and Rice (1975), and Rice (1976) proposed a bifurcation criterion characterized by the singularity of the acoustic tensor, also known as discontinuous bifurcation. It has been shown that this criterion corresponds to the loss of ellipticity of the partial differential equations governing the associated boundary value problem. In the same manner, some authors (Bigoni and Hueckel, 1991; Neilsen and Schreyer, 1993) have suggested the use of the more conservative condition of strong ellipticity, which has been shown to coincide with Rice's criterion within the framework of associative plasticity and small strains. This condition of loss of strong ellipticity is also a special case of Drucker's general bifurcation criterion, in which the bifurcation mode is restricted to localized (compatible) deformation modes.

Finally, a fourth noteworthy set of criteria pertains to the approaches based on stability theory. In these approaches, necking and localization phenomena are addressed within the framework of the stability analysis of local equilibrium equations for a material point. Starting from the general mathematical concept of stability, which was introduced by Lyapunov (1892) and commonly applied to structural instability problems (see, e.g., Abed-Meraim, 1999; Abed-Meraim and Nguyen, 2007), the associated linear perturbation method was extended to material instability problems by Molinari and co-workers (Molinari and Clifton, 1987; Dudzinski and Molinari, 1991). The analysis consists of linearizing the perturbation equations to investigate the growth rate, which is given by the associated eigenvalue problem and will ultimately characterize the stable and unstable modes. Toth et al. (1995) and Boudeau et al. (1998) applied this strategy to micromechanical models. More recently, Li and Karr (2009) adopted this approach to investigate ductile fracture in tension. For strain-rate-dependent materials, this methodology can be viewed as an interesting alternative to bifurcation approaches, which are no longer applicable. At the limit of vanishing rate sensitivity, it was shown that Rice's bifurcation criterion is recovered with this approach (Barbier et al., 1998; Benallal, 2008). 
From this overview of the various approaches pertaining to strain localization criteria and indicators, an interesting observation can be made. While $\mathrm{M}-\mathrm{K}$ analysis has been widely used in the literature, few applications of Rice's ellipticity loss theory, mainly restricted to plane-stress assumptions, particular loading paths, and simple behavior models, have been attempted in sheet metal forming for quantifying metals in terms of their formability. Thus, the main objective of this study is to model plastic instabilities in sheet metals by means of both Rice's bifurcation criterion and the condition of loss of strong ellipticity. Their sound theoretical foundations and fully threedimensional formulation, which allows for the consideration of through-thickness effects (e.g., outof-plane orientation of localization bands), are additional motivations behind our choice of these criteria. Furthermore, in contrast to the $\mathrm{M}-\mathrm{K}$ analysis, whose predictions are overly dependent on the arbitrary choice of the initial defect size, no additional user-defined parameters are required for either Rice's bifurcation or the loss of strong ellipticity. Note that in a recent study by Yoshida and Kuroda (2012a), the bifurcation approach has been used along with the full-constraint Taylor model and the plane-stress assumptions. By contrast, two distinguishing features of the current contribution are: (i) the use of a self-consistent scale-transition scheme, and (ii) the consideration of a fully three-dimensional framework with no plane-stress assumptions. In what follows, strain localization analyses will be carried out on single crystals and polycrystalline aggregates. The micromechanical approach based on crystal plasticity is used to model single crystal behavior, while a self-consistent scale-transition scheme is adopted to derive the polycrystalline behavior.

The remainder of the paper is organized into three main parts.

In the first part, a micromechanical constitutive model based on crystal plasticity is presented. After a literature review of the main contributions devoted to the prediction of defects in sheet metal forming, the single crystal constitutive equations are discussed within the framework of large-strain elasto-plasticity. Then, Hill's self-consistent scale-transition scheme (Hill, 1965a,b) is used to derive the macroscopic behavior of polycrystalline aggregates. In this averaging process, textural and morphological evolutions are taken into account. The internal variables adopted in this constitutive modeling are the dislocation densities per slip system, whose evolution laws account for the mechanisms of dislocation storage and annihilation.

In the second part, the selected localization criteria, namely Rice's bifurcation criterion and the condition of loss of strong ellipticity, are described in some detail. For the sake of clarity, the formulations of these criteria are developed both with respect to the initial undeformed reference configuration and relative to the current configuration. A theoretical relationship between the two criteria is also shown, revealing that one is always slightly more conservative than the other and providing the particular conditions where the two criteria lead to the same predictions. The theoretical classifications of these criteria must be verified through subsequent simulation results. 
In the third part, the previously defined criteria are applied to single crystals with different initial crystallographic orientations and polycrystalline aggregates. More specifically, focus will be placed on the investigation of the effect of microstructural parameters on the ductility limit of metal sheets. Once established, the relationships between microstructure and material formability can be used advantageously to help design new materials with improved formability properties. Such a strategy may be adopted at an early stage of the design of new grades of steel. Finally, the main results are summarized, and some concluding remarks are drawn.

\section{Micromechanical approach based on crystal plasticity}

Sheet metal forming processes may result in various failure modes. For ductile materials, the most common failure modes that limit material formability are strain localization and necking. Localization by shear bands, referred to as material or plastic instability, occurs due to competing hardening and softening mechanisms, while necking may be attributed to the combination of material softening and geometric changes in the specimen. This study focuses on the onset of strain localization in the form of shear band.

Previous investigations that introduced Rice's bifurcation approach into the framework of single crystal plasticity have been presented in the pioneering works of Asaro and Rice (1977), Peirce (1983), and Peirce et al. (1982, 1983). To explicitly derive the expression of a critical hardening modulus, these contributions have simplified the analysis by assuming that only two slip systems can be active. Although this intrinsic criterion can be applied to determine FLDs, no scale-transition scheme has been used to derive the behavior of polycrystalline aggregates, and thus no FLD predictions have been attempted. In more recent studies, single crystal plasticity behavior models have been incorporated into finite element methods to numerically simulate localized necking (Viatkina et al., 2005; Wu et al., 2007; Sun et al., 2009; Zhang et al., 2009). However, mesh sensitivity is a well-known drawback of such FE-based formability analyses (Harewood and McHugh, 2006). It has been shown, nevertheless, that this mesh sensitivity can be removed when using non-local constitutive models, such as those considered in Borg (2007) within the framework of non-local crystal plasticity, in Lele and Anand (2009) within strain-gradient viscoplasticity, or in Mroginski et al. (2011) for porous media.

Among the key factors that influence FLD predictions, the choice of the constitutive model has been shown to have a significant impact on the results (Horstemeyer, 2000). The effect of strainpath changes is often not accounted for in phenomenological constitutive laws; thus, the use of advanced, more predictive behavior models seems to be an interesting way to enhance FLD predictions. An attempt to do this type of investigation can be found in the work of Hiwatashi et al. 
(1998), who applied an advanced anisotropic constitutive model based on texture and dislocation structure to predict FLDs, thus allowing the effect of changes in the strain path to be investigated.

In the last few decades, there has been a growing interest in the use of micromechanical models to predict the ductility limits of polycrystalline aggregates. Boudeau et al. (1998) coupled a micromechanical local law with the Taylor scale-transition scheme to predict FLDs with or without prestrain. In more recent works (Zhou and Neale, 1995; Inal et al., 2005; Wu et al., 2005), a viscoplastic model using the Taylor scale-transition scheme was combined with the M-K analysis to determine FLDs with or without prestrain. Similar FLD analyses with Taylor-based micromechanical models coupled with the M-K approach can be found in Knockaert et al. (2002), Yoshida et al. (2007), and Yoshida and Kuroda (2012b). Recently (Signorelli et al., 2009), significant advances in the FLD analysis have been made by considering a rate-dependent polycrystalline self-consistent plasticity model in conjunction with the $\mathrm{M}-\mathrm{K}$ approach, while previous M-K applications were restricted to the full-constraint Taylor model.

In plastic instability predictions based on crystal plasticity, a key factor is related to the vertex formation on the yield surface. This phenomenon has been pointed out earlier by Hill (1967), and revealed by calculations based on crystal plasticity (Hutchinson, 1970; Kuroda and Tvergaard, 1999). Moreover, experimental evidence of such a yield surface vertex has been shown through carefully designed experiments (Kuwabara et al., 2000). It has been shown that this vertex effect plays a major role both in predictions of material instabilities (Rice, 1976; Hutchinson and Tvergaard, 1981), and also for more realistic predictions of structural instabilities (e.g., plastic buckling; see, Hutchinson (1974), Sewell $(1973,1974))$. In view of this, phenomenological constitutive theories have been developed to include vertex-type effects (Christoffersen and Hutchinson, 1979; Kuroda and Tvergaard, 2001a). In the same way, to overcome the limitations of the flow theory with associative plasticity and smooth yield surface in predicting plastic instabilities, the alternative deformation theory has been proposed in various investigations (see, e.g., Stören and Rice, 1975). The latter theory has been shown to introduce, in an approximate phenomenological way, the vertex effects inherent in physically based micromechanical descriptions. The common feature of these approaches is a reduction in the instantaneous shear moduli (see, e.g., Hutchinson, 1970; Yoshida et al., 2009), which correspondingly reduces the predicted bifurcation levels to realistic values, in the same manner as do deviations from normality in the plastic flow rule (see, e.g., Rudnicki and Rice, 1975; Kuroda and Tvergaard, 2001b).

The main objective here is to predict the ductility limit of polycrystalline materials as accurately as possible by considering the effect of changing the loading paths along with microstructural aspects. Both criteria, namely Rice's bifurcation and the loss of strong ellipticity, will be coupled with a constitutive model derived from a large-strain micromechanical approach and self-consistent 
scale-transition scheme. The choice of such polycrystalline modeling is motivated by the fact that it is now well-known that strain localization predictions are highly sensitive to the formation of vertex effects on the current yield surface and other microstructural mechanisms, such as textural and microstructural anisotropy (see, e.g., Bassani et al., 1978; Neale and Chater, 1980; Barlat, 1987; Lian et al., 1989), which cannot be reproduced by standard phenomenological approaches (see, e.g., Kobayashi, 2010). Thus, because the mechanical characteristics of a material are determined by its microstructure and microscopic properties, a micromechanical model considering the effects of crystallographic texture and those relating to the microstructure and its evolution with changing loading paths would be a better candidate to develop a predictive tool for quantifying the formability of polycrystalline materials. This predictive tool, once validated, could serve in the design process of new grades of steel having improved ductility and in-use properties.

\subsection{Single crystal constitutive modeling}

Restricting our attention to rate-independent behavior with crystallographic slip as the only mechanism of irreversible deformation, the most important aspects of single crystal behavior are the elastic distortion of the lattice and the plastic flow due to slip on the crystallographic planes. The elastic-plastic, finite strain single crystal description adopted here is based on the constitutive theory first developed by Hill (1966) and its subsequent enhanced developments by Asaro (1979, 1983), Nemat-Nasser et al. (1981), and Iwakuma and Nemat-Nasser (1984). A similar formulation has been adopted by Lipinski and Berveiller (1989) and Lipinski et al. (1995). Only the main lines are recalled, and for completeness, the equations that are essential for the subsequent analyses are provided. The starting point is the multiplicative decomposition of the deformation gradient $\mathbf{F}$ as

$$
\mathbf{F}=\mathbf{F}^{e} \cdot \mathbf{F}^{p}
$$

In this decomposition, the intermediate configuration $\mathbf{F}^{p}$ describes the material shear flow along the various slip systems of the crystal, while $\mathbf{F}^{e}$ represents the lattice elastic distortion along with the rigid rotation of the crystal, as illustrated in Fig. 1. The velocity gradient is then written as

$$
\mathbf{g}=\dot{\mathbf{F}} \cdot \mathbf{F}^{-1}=\dot{\mathbf{F}}^{e} \cdot \mathbf{F}^{e-1}+\mathbf{F}^{e} \cdot\left(\dot{\mathbf{F}}^{p} \cdot \mathbf{F}^{p-1}\right) \cdot \mathbf{F}^{e-1}
$$

in which

$$
\mathbf{F}^{e} \cdot\left(\dot{\mathbf{F}}^{p} \cdot \mathbf{F}^{p-1}\right) \cdot \mathbf{F}^{e-1}=\mathbf{d}^{p}+\mathbf{w}^{p}=\sum_{g} \dot{\gamma}^{g} \overrightarrow{\mathbf{m}}^{g} \otimes \overrightarrow{\mathbf{n}}^{g}
$$

where $\overrightarrow{\mathbf{m}}^{g}$ is the vector located in the slip direction of the slip plane $g$ with normal $\overrightarrow{\mathbf{n}}^{g}, \dot{\gamma}^{g}$ is the associated slip rate, and $\mathbf{d}^{p}$ and $\mathbf{w}^{p}$ are the plastic strain rate and plastic spin, respectively. In the same way, $\dot{\mathbf{F}}^{e} \cdot \mathbf{F}^{e-1}$ is split into its symmetric part $\mathbf{d}^{e}$ and its anti-symmetric part $\mathbf{w}^{e}$ as follows:

$$
\dot{\mathbf{F}}^{e} \cdot \mathbf{F}^{e-1}=\mathbf{d}^{e}+\mathbf{w}^{e}
$$


so that

$$
\mathbf{g}=\mathbf{d}+\mathbf{w}, \quad \mathbf{d}=\mathbf{d}^{e}+\mathbf{d}^{p}, \quad \mathbf{w}=\mathbf{w}^{e}+\mathbf{w}^{p}
$$

where $\mathbf{d}$ and $\mathbf{w}$ are the symmetric and anti-symmetric parts of $\mathbf{g}$, respectively. From Eq. (3), the plastic strain rate $\mathbf{d}^{p}$ and plastic spin $\mathbf{w}^{p}$ can be written in terms of the Schmid tensors $\mathbf{R}^{g}$ and $\mathbf{S}^{g}$, defined as the symmetric and anti-symmetric parts, respectively, of the tensor product $\overrightarrow{\mathbf{m}}^{g} \otimes \overrightarrow{\mathbf{n}}^{g}$.

If $\boldsymbol{\sigma}$ denotes the Cauchy stress tensor and $\boldsymbol{\tau}=J \boldsymbol{\sigma}$ is the Kirchhoff stress tensor, with $J=\operatorname{det} \mathbf{F}$, then, as observed by Hill (1966), it is natural to use stress rates that are co-rotational with the elastic distortion of lattice to describe the local elastic response. This particular objective derivative reads

$$
\boldsymbol{\tau}^{\nabla}=\dot{\boldsymbol{\tau}}-\mathbf{w}^{e} \cdot \boldsymbol{\tau}+\boldsymbol{\tau} \cdot \mathbf{w}^{e}
$$

The local stress variation can then be written as follows (see, e.g., Hill and Rice, 1972):

$$
\boldsymbol{\tau}^{\nabla}=\mathbf{C}: \mathbf{d}^{e}
$$

where $\mathbf{C}$ is the fourth-order tensor of the elastic constants. Adopting in what follows the framework of an updated Lagrangian approach (i.e., $\mathbf{F}=\mathbf{1}, J=1$ ), the elasticity law (7) becomes

$$
\boldsymbol{\sigma}^{\nabla}+\boldsymbol{\sigma} \operatorname{tr}(\mathbf{d})=\mathbf{C}: \mathbf{d}^{e}
$$

To determine the crystallographic slip rates $\dot{\gamma}^{g}$, the Schmid law is used, leading to a normality rule (Hill, 1966). If the resolved shear stress $\tau^{g}$ acting on a given slip system $g$ is defined as the projection of the Cauchy stress on the Schmid tensor $\mathbf{R}^{g}$ associated with that slip system, such that

$$
\tau^{g}=\boldsymbol{\sigma}: \mathbf{R}^{g}=\left(\boldsymbol{\sigma} \cdot \overrightarrow{\mathbf{n}}^{g}\right) \cdot \overrightarrow{\mathbf{m}}^{g}
$$

then this resolved shear stress appears as a thermodynamic driving force that must exceed a certain threshold of slip resistance so that the associated dislocation densities can move. The threshold for each slip system represents the current yield stress, denoted as the critical resolved shear stress $\tau_{c}^{g}$. For practical reasons and for handling only positive values of slip rates, the total number of slip systems is doubled by distinguishing between pairs $\left(\overrightarrow{\mathbf{m}}^{g}, \overrightarrow{\mathbf{n}}^{g}\right)$ and $\left(-\overrightarrow{\mathbf{m}}^{g}, \overrightarrow{\mathbf{n}}^{g}\right)$. Consequently, the yield function, $f^{g}=\tau^{g}-\tau_{c}^{g}$, for a given slip system, $g$, defines a plasticity criterion given by

$$
f^{g}=\left(\tau^{g}-\tau_{c}^{g}\right) \leq 0, \quad \dot{\gamma}^{g} \geq 0 \quad \text { and } \quad \dot{\gamma}^{g} f^{g}=0
$$

These inequalities indicate that there is no plastic slip in this slip system (i.e., $\dot{\gamma}^{g}=0$ ) if the stress is located in the interior of the yield surface $\left(f^{g}<0\right)$ or unloading occurs from the yield surface ( $f^{g}=0$ and $\dot{f}^{g}<0$ ). However, strict plastic flow (i.e., $\left.\dot{\gamma}^{g}>0\right)$ implies that $f^{g}=0$ and $\dot{f}^{g}=0$.

To proceed further with the determination of the slip rates, we must use the consistency condition. The latter $\left(\dot{f}^{g}=\dot{\tau}^{g}-\dot{\tau}_{c}^{g}=0\right)$ involves the derivative of the Schmid tensors, which are embedded in the crystallographic lattice. Assuming that the elastic strains remain small compared to 
unity, the evolution laws of Schmid's vectors can be approximated as $\dot{\overrightarrow{\mathbf{m}}}^{g}=\mathbf{w}^{e} \cdot \overrightarrow{\mathbf{m}}^{g}$ and $\dot{\overrightarrow{\mathbf{n}}}^{g}=\mathbf{w}^{e} \cdot \overrightarrow{\mathbf{n}}^{g}$ (Anand and Kothari, 1996), leading to the following relation for the resolved shear stress rate:

$$
\dot{\tau}^{g}=\overrightarrow{\mathbf{m}}^{g} \cdot \dot{\boldsymbol{\sigma}} \cdot \overrightarrow{\mathbf{n}}^{g}+\dot{\overrightarrow{\mathbf{m}}}^{g} \cdot \boldsymbol{\sigma} \cdot \overrightarrow{\mathbf{n}}^{g}+\overrightarrow{\mathbf{m}}^{g} \cdot \boldsymbol{\sigma} \cdot \dot{\overrightarrow{\mathbf{n}}}^{g}=\overrightarrow{\mathbf{m}}^{g} \cdot \boldsymbol{\sigma}^{\nabla} \cdot \overrightarrow{\mathbf{n}}^{g}=\boldsymbol{\sigma}^{\nabla}: \mathbf{R}^{g}
$$

In summary, in our modeling, the orientation of the crystallographic frame is defined by the classical Euler angles and their evolution equations with the elastic lattice spin $\mathbf{w}^{e}$ (see, e.g., Bunge, 1982; Lipinski et al., 1995). These equations are detailed in Franz et al. (2009a) for the evolution of both crystallographic and morphological orientations. The latter is defined by the orientation of the principal frame of the ellipsoid representing the grain, whose shape evolution is also taken into account by the evolution equations of the ellipsoid half-axes.

The last two points that complete the presentation of the elastic-plastic single crystal constitutive modeling are: (i) the evolution laws of hardening, and (ii) the procedure of active slip system selection. Before discussing the non-trivial issue of active slip system selection, we assume that these systems are known and first determine their slip rates and the single crystal tangent modulus. Assume that the evolution of the critical resolved shear stress is given by a law of the form

$$
\dot{\tau}_{c}^{g}=\sum_{h} H^{g h} \dot{\gamma}^{h}
$$

in which summation is over the active slip systems, and the hardening interaction matrix $H^{g h}$ will be described later. Combining the consistency condition with Eqs. (3), (5), (8), (11), and (12) yields

$$
\sum_{h}\left(H^{g h}+\mathbf{R}^{g}: \mathbf{C}: \mathbf{R}^{h}\right) \dot{\gamma}^{h}=\mathbf{R}^{g}:(\mathbf{C}-\boldsymbol{\sigma} \otimes \mathbf{1}): \mathbf{d}
$$

This resulting linear system involves a square matrix whose size is equal to the current number of active slip systems, and whose $(g, h)$ th component is $\left(H^{g h}+\mathbf{R}^{g}: \mathbf{C}: \mathbf{R}^{h}\right)$. If $M^{g h}$ stands for the $(g, h)$ th component of the inverse of this matrix, then the slip rates are obtained as

$$
\dot{\gamma}^{g}=\sum_{h} M^{g h} \mathbf{R}^{h}:(\mathbf{C}-\boldsymbol{\sigma} \otimes \mathbf{1}): \mathbf{d}
$$

Within the finite-strain framework, several tangent moduli can be chosen depending on the selected stress measure. The single crystal tangent modulus sought here takes the following form:

$$
\dot{\mathbf{n}}=\mathbf{l}: \mathbf{g}
$$

where $\mathbf{n}$ is the nominal stress, which is related to the Cauchy stress by $\mathbf{n}=J \mathbf{F}^{-1} \cdot \boldsymbol{\sigma}$.

Taking the current configuration as a reference configuration, one obtains

$$
\begin{aligned}
\dot{\mathbf{n}}= & \mathbf{C}: \mathbf{g}-{ }_{\boldsymbol{\sigma}}^{1} \mathbf{L}: \mathbf{g}-{ }_{\boldsymbol{\sigma}}^{2} \mathbf{L}: \mathbf{g} \\
& -\sum_{g, h}\left(\mathbf{C}: \mathbf{R}^{g}+\mathbf{S}^{g} \cdot \boldsymbol{\sigma}-\boldsymbol{\sigma} \cdot \mathbf{S}^{g}\right) M^{g h}\left(\mathbf{R}^{h}: \mathbf{C}-\mathbf{R}^{h}: \boldsymbol{\sigma} \otimes \mathbf{1}\right): \mathbf{g}
\end{aligned}
$$

where the fourth-order tensors ${ }_{\sigma}^{1} \mathbf{L}$ and ${ }_{\sigma}^{2} \mathbf{L}$ contain the convective terms of the stress components, as explicitly shown by the expression of the tangent modulus $\mathbf{l}$ of Eq. (15) in indicial notation 


$$
\begin{aligned}
l_{i j k l}= & C_{i j k l}-\frac{1}{2}\left(\sigma_{i k} \delta_{j l}-\sigma_{i l} \delta_{j k}\right)-\frac{1}{2}\left(\delta_{i k} \sigma_{j l}+\delta_{i l} \sigma_{j k}\right) \\
& -\sum_{g, h}\left(C_{i j m n} R_{m n}^{g}+S_{i m}^{g} \sigma_{m j}-\sigma_{i m} S_{m j}^{g}\right) M^{g h}\left(R_{p q}^{h} C_{p q k l}-R_{p q}^{h} \sigma_{p q} \delta_{k l}\right)
\end{aligned}
$$

in which it is clear that this tangent modulus does not have any of the minor or major symmetries.

Returning to the active slip system selection, which has not been addressed thus far, it should be noted that there are two difficulties associated with this issue. The first concerns the evaluation of the $M^{g h}$ components due to possible singularity of matrix $N^{g h}=\left(H^{g h}+\mathbf{R}^{g}: \mathbf{C}: \mathbf{R}^{h}\right)$. For instance, some simplified hardening rules proposed in the early approaches resulted in equality between two or several columns of matrix $N^{g h}$, making it non-invertible. This issue has been widely discussed in the literature, and several remedies have been proposed to circumvent this difficulty. With the hardening laws adopted in the current constitutive modeling, we have not encountered this problem; however, we did face the second difficulty inherent to rate-independent approaches. This issue is related to the non-uniqueness of the set of active slip systems. This difficulty can be understood by observing the nature of Eq. (13), in which one must solve a linear system in which the dimension of the matrix to be inverted is itself unknown. In other words, this difficulty is due to the fact that the consistency condition for a given slip system $g, \dot{\tau}^{g}=\dot{\tau}_{c}^{g}$, cannot be independently solved because the hardening interaction terms require coupling with other active slip systems. In the literature, the concept of potentially active $\left(\tau^{g}=\tau_{c}^{g}\right)$ and effectively active $\left(\tau^{g}=\tau_{c}^{g}\right.$ and $\left.\dot{\tau}^{g}=\dot{\tau}_{c}^{g}\right)$ slip systems was introduced, and it was shown that, within the potentially active systems, several subsets of active systems may exist. This issue has been discussed in several reports (Hill and Rice, 1972; Franciosi, 1984; Franciosi and Zaoui, 1991; Anand and Kothari, 1996; Busso and Cailletaud, 2005; Arul Kumar and Mahesh, 2012), and various methods have been proposed to address this indetermination issue. Franciosi and Zaoui (1991) suggested an energy-based criterion for the selection of active slip systems: within the potentially active systems, the combination of slip systems that is chosen to be active is the one that minimizes the deformation energy. From a computational point of view, this criterion requires a combinatory analysis to be performed for each time step, which is extremely time consuming.

In our approach, a new method is proposed to determine the active slip systems and their slip rates within an elastic-plastic modeling framework. This method, inspired by viscoplastic formulations, allows the computing time to be considerably reduced while preserving the rateindependent nature of the constitutive equations. The starting point consists of setting the plastic flow rule for a given slip system $g$ in the following equivalent form: 


$$
\left\{\begin{array}{l}
\tau^{g}<\tau_{c}^{g} \Rightarrow \dot{\gamma}^{g}=0 \\
\tau^{g}=\tau_{c}^{g} \text { and } \dot{\tau}^{g} \leq 0 \Rightarrow \dot{\gamma}^{g}=0 \\
\tau^{g}=\tau_{c}^{g} \text { and } \dot{\tau}^{g}>0 \Rightarrow \dot{\gamma}^{g} \geq 0
\end{array}\right.
$$

Then, regularization of these equations is proposed to model a threshold (or step function) with a smoother function, thus circumventing the computational issues inherent in the use of stiff functions. Several regularization techniques are possible; we adopt the following form:

$$
\dot{\gamma}^{g}=k^{g}\left(\tau^{g}, \tau_{c}^{g}, \dot{\tau}^{g}\right) \dot{\tau}^{g}
$$

in which the regularization function $k^{g}$ is chosen to have the following expression:

$$
k^{g}=\frac{1}{H^{g g}} \frac{1}{2}\left[1+\tanh \left(k_{0} \frac{\tau^{g}}{\tau_{r e f}}\right)\right] \frac{1}{2}\left[1+\tanh \left(k_{1}\left\{\frac{\tau^{g}}{\tau_{c}^{g}}-1\right\}\right)\right] \frac{1}{2}\left[1+\tanh \left(k_{2} \frac{\dot{\tau}^{g}}{\dot{\tau}_{\text {ref }}}\right)\right]
$$

where ' tanh' denotes the hyperbolic tangent function, and $k_{0}, k_{1}, k_{2}, \tau_{\text {ref }}$, and $\dot{\tau}_{\text {ref }}$ are numerical parameters associated with the regularization function (see Franz et al., 2009a). Combining this regular form of Schmid's law (19) with Eqs. (11) and (8), and using Eqs. (3) and (5), one obtains

$$
\sum_{h}\left(\delta^{g h}+\mathbf{R}^{\prime g}: \mathbf{C}: \mathbf{R}^{h}\right) \dot{\gamma}^{h}=\mathbf{R}^{\prime g}:(\mathbf{C}-\mathbf{\sigma} \otimes \mathbf{1}): \mathbf{d}
$$

where $\mathbf{R}^{\prime g}=k^{g} \mathbf{R}^{g}$ (with no summation over $g$ ), and $\delta^{g h}$ are the components of the identity matrix, whose size is the current number of active slip systems. The slip rates are then given by

$$
\dot{\gamma}^{g}=\sum_{h} P^{g h} \mathbf{R}^{\prime h}:(\mathbf{C}-\boldsymbol{\sigma} \otimes \mathbf{1}): \mathbf{d}
$$

where $P^{g h}$ represents the $(g, h)$ th component of the inverse of the matrix whose components are $Q^{g h}=\delta^{g h}+\mathbf{R}^{\prime g}: \mathbf{C}: \mathbf{R}^{h}$. Note that Eqs. (21)-(22) are the counterparts of Eqs. (13)-(14) once regularization is applied. This regularization also affects the expression of the tangent modulus given in Eq. (17). Repeating the same steps as before, with the new expression of the slip rates given by Eq. (22), the expression of the tangent modulus in indicial notation becomes

$$
\begin{aligned}
l_{i j k l}= & C_{i j k l}-\frac{1}{2}\left(\sigma_{i k} \delta_{j l}-\sigma_{i l} \delta_{j k}\right)-\frac{1}{2}\left(\delta_{i k} \sigma_{j l}+\delta_{i l} \sigma_{j k}\right) \\
& -\sum_{g, h}\left(C_{i j m n} R_{m n}^{g}+S_{i m}^{g} \sigma_{m j}-\sigma_{i m} S_{m j}^{g}\right) P^{g h} R_{p q}^{\prime h}\left(C_{p q k l}-\sigma_{p q} \delta_{k l}\right)
\end{aligned}
$$

Note the similarity in the algebraic structure of the tangent moduli given by Eqs. (17) and (23), the latter being affected by regularization through the components $P^{g h}=\left(\delta^{h g}+k^{h} \mathbf{R}^{h}: \mathbf{C}: \mathbf{R}^{g}\right)^{-1}$ and $R_{p q}^{\prime h}=k^{h} R_{p q}^{h}$, in which the regularization function $k^{h}$ is given by Eq. (20). Once again, the resulting tangent modulus (23) has none of the minor or major symmetries. 
For validation purposes, it has been verified that the results obtained with this regularization procedure are quasi-identical to the reference results given by the conventional slip system selection methods (see Franz et al. (2009a,b) for more details).

Note that other approaches in the literature round off the corners of the yield surface, which results in a regular form of Schmid's law and eliminates the ambiguity in the determination of active slip systems (Arminjon 1991; Gambin, 1991, 1992). See also Peeters et al. (2001) for an anisotropy-based approach. Although these regularization methods result in a slight deviation from Schmid's law, this deviation is outweighed by their benefits in terms of computational efficiency.

Similarly to other approaches, the regularization method that we proposed also has parameters whose values need to be determined. To this end, a parametric study has been carried out to model a threshold (step function) while avoiding the numerical problems inherent to the use of sharp functions (Lorrain, 2005; Franz, 2008). Comparisons with reference results pertaining to active slip systems for single crystals with different initial orientations (Nesterova et al., 2001) have demonstrated the validity of the proposed methodology (Franz et al., 2009a).

Finally, the single crystal hardening law allows the evolution of critical resolved shear stresses to be expressed in terms of slip rates of active slip systems by introducing the hardening matrix that defines self-hardening and latent hardening. The expression of such a law, already anticipated through Eq. (12), requires a description of hardening based on dislocation interactions in relation to their creation, storage and annihilation (Franciosi, 1984; Tabourot, 1992), as shown below:

$$
\tau_{c}^{g}=\tau_{0}^{g}+\alpha \mu b \sqrt{\sum_{h=1}^{n_{g l}} a^{g h} \rho^{h}}
$$

where $\tau_{0}^{g}$ is the initial critical shear stress, $\alpha$ is a constant related to the stability of the dislocation configurations, $\mu$ is the shear modulus, $b$ is the magnitude of the Burgers vector, $a^{g h}$ is the anisotropy interaction matrix, and $\rho^{h}$ is the mean dislocation density for slip system $h$. The anisotropy interaction matrix introduced by Franciosi (1984) and expanded by Hoc (1999) will be used, in which the different components are defined by nine parameters depending on the nature of the dislocation interactions (e.g., coplanar or collinear systems...).

The evolution of the dislocation densities without a specified annihilation mechanism has been modeled by Kocks (1976); the annihilation of close dislocations was first considered by Essmann and Mughrabi (1979), leading to a law of the following form:

$$
\dot{\rho}^{g}=\frac{1}{b}\left(\frac{1}{L^{g}}-2 y_{c} \rho^{g}\right) \dot{\gamma}^{g}
$$

where $y_{c}$ is the critical annihilation distance of the dislocations and $L^{g}$ is the mean free path of the dislocations on the slip system $g$ given by 


$$
\frac{1}{L^{g}}=\frac{1}{D}+\frac{\sqrt{\sum_{h=1, h \neq g}^{n_{g l}} \rho^{h}}}{g_{0}}
$$

where $D$ represents the average grain size and $g_{0}$ corresponds to a parameter related to the dislocation storage. Note that Eq. (26) suggests that the value to assign to parameter $D$ should depend, in the case of single crystals, on whether the specimen size is larger or smaller than the maximum mean free path of dislocations; while for polycrystalline aggregates, this should depend on whether the average grain size is larger or smaller than the maximum mean free path of dislocations. Accordingly, for single crystals, $D$ will be given the value of the specimen size if the latter is larger than the maximum mean free path of dislocations; otherwise, $D$ will be given the value of the maximum mean free path of dislocations. For polycrystalline aggregates, $D$ will be given the value of the average grain size if the latter is larger than the maximum mean free path of dislocations; otherwise, $D$ will be given the value of the maximum mean free path of dislocations. Differentiating Eq. (24) and using Eq. (25), the hardening matrix is expressed as

$$
H^{g h}=\frac{\alpha \mu}{2 \sqrt{\sum_{k=1}^{n_{g l}} a^{g k} \rho^{k}}} a^{g h}\left(\frac{1}{L^{h}}-2 y_{c} \rho^{h}\right)
$$

For the time integration of the single crystal constitutive equations developed in this section, several explicit time integration schemes of different orders have been assessed. Based on this comparison, we have selected the fourth-order Runge-Kutta algorithm, which is more accurate than the other explicit schemes tested (i.e., forward Euler and second-order Runge-Kutta). Compared to iterative implicit schemes, this choice has been motivated by its straightforward implementation and the intended applications within the current study. Furthermore, this choice is particularly justified for the simulation of linear or sequential loading paths and for localization analyses along such simple loading paths. To preserve the accuracy of the simulation results, the loading increments are kept sufficiently small and an adaptive time step is used during abrupt stiffness changes, which correspond to the transition from an elastic regime to a plastic regime when changing loading paths.

Note that the above model has been validated by comparison with results reported in the literature (Lorrain, 2005; Franz et al., 2009a). These results correspond to different Fe and Fe-Si single crystals with a cubic centered structure, various initial orientations, and undergoing linear strain paths (tensile test, shear test ...) and sequential loading paths (Bauschinger test) (Rauch, 1998; Keh and Nakada, 1967; Pollnow et al., 1972). For each material, the initial orientation given by the Euler angles is required, in addition to four material parameters, the average grain size $D$, parameter $g_{0}$, which is related to the mean free path of dislocations, the critical annihilation distance of dislocations $y_{c}$, and the initial critical shear stress $\tau_{0}^{g}$. For single crystals, comparisons between the simulation results and experiments have been found to have good agreement for a wide range of linear and sequential loading paths and various initial orientations (Lorrain, 2005). 


\subsection{Self-consistent scale transition and polycrystalline behavior}

The aim of the scale transition is to estimate the overall behavior of polycrystalline aggregates from knowledge of the behavior of their individual constituents. The scale transition is typically performed using mean field approaches to seek approximations for the instantaneous moduli of polycrystals as functions of their constituent grains moduli. These averaging processes also seek to account for interactions between the grain and its neighborhood within the polycrystal, which must result in a compatible overall deformation.

A significant amount of work has been dedicated to this subject (Voigt, 1889; Sachs, 1928; Reuss, 1929; Boas and Schmid, 1934; Taylor, 1934, 1938). In Taylor (1938), a scheme for rigidplastic models was proposed, which was later generalized by Bishop and Hill (1951a,b). Lin's approach (Lin, 1957, 1971) generalized Taylor's scheme by including elasticity effects. The development of self-consistent schemes (Hershey, 1954; Kröner, 1958, 1961; Budiansky and Wu, 1962) and the key contributions of Budiansky (1965) and Hill (1965a,b) were significant advancements in the field. A detailed presentation of three major averaging techniques, attributed to Lin, Budiansky-Kröner-Wu and Hill, was given by Hutchinson (1970), who showed that Hill's self-consistent scheme yields a less stiff overall response. The development of this self-consistent scheme was pursued in more recent contributions by Berveiller and Zaoui (1979) and Weng (1980).

All of the descriptions discussed above pertain to the small-strain framework. The case of polycrystals undergoing large strains was considered later with the fundamental work of Hill (1972), which established the adequate framework and proposed the pioneering directions. Within large-strain elasto-plasticity, the shape of individual grains changes with plastic flow, and the lattice orientation is affected by rotations resulting in texture development. Iwakuma and Nemat-Nasser (1984) proposed a general large-strain elastic-plastic formulation for polycrystalline aggregates composed of single crystals deforming by lattice distortion and crystallographic slip. This approach is based on the averaging theorems proposed by Hill and on the calculation of Green's functions for incremental strains applied to a polycrystal. In this contribution, the rate problem is formulated in terms of the nominal stress rate, which allows for the estimation of the associated instantaneous moduli linking the overall nominal stress rate to the corresponding macroscopic velocity gradient. Note that the application of this description (Iwakuma and Nemat-Nasser, 1984) was restricted to the case of double slip (i.e., only two active slip systems are considered) for polycrystals under uniaxial loading. These approaches were further developed by Lipinski and Berveiller (1989), who did not place any restrictions on the number of active slip systems. In the current study, this wellknown scheme will be used to derive the macroscopic behavior from knowledge of the behavior at the microscale and the microstructure (including texture) of the representative volume element. 
Only the main points are outlined below, and the detailed derivations can be found in earlier references (Lipinski and Berveiller, 1989; Lipinski et al., 1995; Franz et al., 2009a).

As mentioned before, efforts to formulate the local behavior in terms of adequate work conjugate variables (nominal stress rate - velocity gradient) will simplify the scale transition. As suggested by Hill (1972), this choice reduces the homogenization procedures to simple volume average rules, similarly to the small-strain framework. In addition, due to the implicit and incremental nature of the integral equation resulting from this homogenization scheme, the hypoelastic form of the local constitutive law is particularly well adapted. By expressing the macroscopic behavior law, linking the macroscopic nominal stress rate $\dot{\mathbf{N}}$ to the macroscopic velocity gradient $\mathbf{G}$ via the macroscopic tangent modulus $\mathbf{L}$, in the same incremental form as that of the single crystal (15), one obtains

$$
\dot{\mathbf{N}}=\mathbf{L}: \mathbf{G}
$$

where the macroscopic fields are defined as the volume averages of their microscopic counterparts:

$$
\left\{\begin{array}{l}
\mathbf{G}=\frac{1}{V} \int_{V} \mathbf{g}(\mathbf{x}) d v=\overline{\mathbf{g}(\mathbf{x})} \\
\dot{\mathbf{N}}=\frac{1}{V} \int_{V} \dot{\mathbf{n}}(\mathbf{x}) d v=\overline{\dot{\mathbf{n}}(\mathbf{x})}
\end{array}\right.
$$

Then, the following fourth-order concentration tensor must be introduced to obtain a systematic expression of the macroscopic tangent modulus:

$$
\mathbf{g}(\mathbf{x})=\mathbf{A}(\mathbf{x}): \mathbf{G}
$$

Under these conditions, it is easy to show that the macroscopic tangent modulus is given by

$$
\mathbf{L}=\frac{1}{V} \int_{V} \mathbf{l}(\mathbf{x}): \mathbf{A}(\mathbf{x}) d v=\overline{\mathbf{l}(\mathbf{x}): \mathbf{A}(\mathbf{x})}
$$

At this stage, it is commonly assumed that for each individual grain, the behavior and mechanical fields are homogeneous. For a given grain $I$ of volume $V^{I}$, an indicator function $\theta^{I}$ is defined by

$$
\left\{\begin{array}{lll}
\theta^{I}(\mathbf{x})=1 & \text { if } & \mathbf{x} \in V^{I} \\
\theta^{I}(\mathbf{x})=0 & \text { if } & \mathbf{x} \notin V^{I}
\end{array}\right.
$$

leading to

$$
\left\{\begin{array}{l}
\mathbf{g}(\mathbf{x})=\sum_{I=1}^{N g} \mathbf{g}^{I} \quad \theta^{I}(\mathbf{x}) \\
\mathbf{l}(\mathbf{x})=\sum_{I=1}^{N g} \mathbf{l}^{I} \theta^{I}(\mathbf{x})
\end{array}\right.
$$

where $\mathbf{g}^{I}$ and $\mathbf{l}^{I}$ are the volume average for grain $I$ of the velocity gradient and the tangent modulus, respectively, and $\mathrm{Ng}$ is the number of grains within the polycrystalline aggregate. 
The derivation of the concentration tensor $\mathbf{A}$ is not a trivial task and requires rather elaborate mathematical development. To this end, detailed formulations can be found in the literature (Berveiller and Zaoui, 1979; Fassi-Fehri, 1985; Berveiller et al., 1987; Lipinski et al., 1995), and only the main lines are summarized below. First, field equations (quasi-static equilibrium, compatibility and boundary conditions) are combined, making use of Green's tensor techniques to transform the problem into an integral equation; see Dederichs and Zeller (1973) and Berveiller and Zaoui (1984) for the small-strain framework and Lipinski and Berveiller (1989) for finite deformations. In that process, a reference fictitious homogeneous medium is introduced. Note that within the large-strain framework, the Lamé operator involved in these derivations is not selfadjoint due to the lack of symmetry of the local and overall tangent moduli. Nevertheless, the construction of the integral formulation is still possible using the adjoint operator of the Green tensor, as suggested by Willis (1988). From these formal developments, and to obtain expressions that are of practical use, the remaining derivations lie in the appropriate choice for the reference homogeneous medium and some simplifications for the integral equation, such as the so-called Born approximation. Finally, by decomposing the modified Green tensor into local and non-local parts and employing some convenient properties (Lipinski and Berveiller, 1989; Lipinski et al., 1995), the concentration tensor $\mathbf{A}^{I}$ for grain $I$ can be written as

$$
\mathbf{A}^{I}=\left(\mathbf{I}-\mathbf{T}^{I I}:\left(\mathbf{l}^{I}-\mathbf{L}\right)\right)^{-1}:{\overline{\left(\mathbf{I}-\mathbf{T}^{I I}:\left(\mathbf{l}^{I}-\mathbf{L}\right)\right)^{-1}}}^{-1}
$$

where $\mathbf{T}^{I I}$ is the interaction tensor for grain $I$, which is related to Eshelby's tensor (Eshelby, 1957) for an ellipsoidal inhomogeneity. Explicit expressions for tensor $\mathbf{T}^{I I}$ can only be found for isotropic media; for general anisotropy, such as in the proposed model, an integral over the ellipsoid is obtained using Fourier's transforms (Fassi-Fehri, 1985; Berveiller et al., 1987), and its numerical evaluation is achieved by means of Gauss-Legendre quadrature. For a polycrystalline aggregate comprising $\mathrm{Ng}$ grains with a respective volume fraction $f^{I}$, the one-site self-consistent expression corresponding to the self-consistent scheme in the sense of Hill (1965a) can be finally obtained as

$$
\mathbf{L}=\sum_{I=1}^{N g} f^{I} \mathbf{l}^{I}: \mathbf{A}^{I}
$$

The expression of this effective modulus as presented in Eqs. (34) and (35) reveals an implicit character because $\mathbf{T}^{I I}, \Delta \mathbf{I}^{I}=\left(\mathbf{l}^{I}-\mathbf{L}\right)$ and thus $\mathbf{A}^{I}$ depend on the yet-unknown modulus $\mathbf{L}$. An iterative procedure is required to conjointly solve Eqs. (34) and (35). In our computational procedure, we adopted an iterative scheme that converges within a few iterations.

Note that the classical full-constraint Taylor model can be derived as a special case of the selfconsistent scheme by considering that the deformation within each grain is equal to the macroscopic 
deformation. This amounts to taking a concentration tensor equal to the fourth-order identity tensor, which leads to an effective modulus simply given by the volume average of the microscopic moduli.

In the same manner as for the time integration of the local constitutive law discussed in the previous section, much attention was paid to computational considerations to avoid affecting the accuracy of this advanced physically based model through its numerical implementation. The choice of the explicit fourth-order Runge-Kutta algorithm for the time integration of the local constitutive law was motivated by a compromise in terms of efficiency, accuracy, and convergence.

These important considerations related to the time integration of constitutive equations have been widely investigated in the literature (Hughes, 1984; Simo and Taylor, 1985; Ortiz and Popov, 1985; Kim et al., 2008; Sánchez et al., 2008; Becker, 2011), and the respective benefits and drawbacks of various implicit and explicit time integration schemes in terms of accuracy, stability, robustness, and efficiency have been discussed. For constitutive equations with numerous internal variables, implicit integration procedures are often avoided, and explicit schemes are adopted instead. In such situations, implicit schemes require a large linear algebraic system to be solved by means of successive iterations involving the inversion of large matrices, which can substantially affect the efficiency and even the convergence. A previous study on the time integration of advanced elasticplastic constitutive models with large numbers of internal variables, yet phenomenological (Haddag et al., 2007), confirms that adequate alternatives would involve either reducing the size of the nonlinear system to be solved (whenever possible) or adopting an explicit time integration scheme.

A last point in this section concerns the evaluation of the self-consistent scheme presented above and the validation of the developed polycrystalline model. This validation was conducted on a selection of single-phase ferritic and dual-phase ferritic-martensitic steels having significantly different microstructures and mechanical characteristics. The experimental results that served as references for comparison were provided by ArcelorMittal. The complete data (chemical compositions, mechanical characteristics, etc) for these steels can be found in Lorrain (2005) and Franz (2008). For representativeness, a few thousand grains are considered systematically, with their initial crystallographic orientations obtained by means of the orientation distribution function (ODF). As mentioned previously, the identification of four parameters relative to the single crystal modeling is required (the initial critical shear stress $\tau_{0}^{g}$, parameter $g_{0}$, which is related to the mean free path of dislocations, the critical annihilation distance of dislocations $y_{c}$, and the average grain size $D$ ). The average grain size can be easily identified using optical micrography. To identify the three other parameters, two mechanical tests are used: a uniaxial tensile test or simple shear test and a reverse shear test. The validation campaign was carried out on a wide range of tests, including rheological stress-strain responses, yield surfaces, texture evolution, and Lankford coefficients. The available mechanical tests included direct strain paths (uniaxial tensile test, simple shear test, plane 
strain tensile test, biaxial tensile test, and balanced biaxial tensile test) in different directions with respect to the rolling direction, as well as sequential strain paths (i.e., Bauschinger test, orthogonal test ...). Comparisons in terms of the stress-strain responses, yield surfaces, texture evolution, and Lankford coefficients have shown good agreement between the experimental and modeling results. For conciseness, only some of the illustrative validation examples will be shown in the subsequent sections, which are dedicated to the application of the model and a discussion of the results. The complete validation can be found in Lorrain (2005) and Franz (2008).

\section{Rice's bifurcation approach and the criterion of loss of strong ellipticity}

In this section, the Rice localization criterion is first developed based on the bifurcation approach by providing the main lines of its formulation, the underlying assumptions, and the various tangent moduli necessary for its application. Unlike most of the available reports, in this study, we choose to express this criterion with respect to a fixed reference configuration and only adopt the updated Lagrangian approach once the criterion is completely established. Such a presentation is believed to afford more clarity, and it allows more flexibility in terms of application, as the reference configuration can be chosen rather freely. This choice is also motivated by the adopted large-strain framework, for which the expression of equilibrium equations relative to a fixed configuration is more suitable to bifurcation analyses because the rate (incremental) form of the equilibrium equations can then be obtained in a more systematic manner.

\subsection{Rice's localization criterion}

The theoretical foundations of this approach were established early on by Hadamard (1903) for elastic solids and then extended to elastic-plastic solids by Thomas (1961), Hill (1962) and Mandel (1966). In the approach adopted by Hill (1962), the conditions under which the equations governing the rate boundary value problem cease to be elliptic are sought (see also Hill and Hutchinson, 1975; Needleman and Tvergaard, 1977). Equivalently, we seek the possibility of bifurcation from a homogeneous deformation state towards a state that overlaps a localization band. As specified by Rudnicki and Rice (1975), this condition corresponds to the onset or incipience of bifurcation.

Let us consider a solid subjected to a loading path corresponding to a homogeneous deformation state. The kinematics and equilibrium equations will be first described relative to a fixed reference configuration that may represent the initial state (see, e.g., Saje et al., 1982); the case where the reference configuration is taken to be equal to the current state will be discussed later (see, e.g., Yamamoto, 1978). The appropriate work conjugate variables are then the Lagrangian gradient of deformation $\mathbf{F}$ and the first Piola-Kirchhoff stress tensor B (defined here following Truesdell and 
Noll (1965), i.e., $\mathbf{B}=\mathbf{N}^{T}$; while its definition in Malvern (1969) is the transpose of that adopted here). Assume that at a certain point along the loading path, the homogeneous fundamental path allows a bifurcation for the first time. At this incipient bifurcation state, the mechanical fields $\mathbf{B}$ and $\mathbf{F}$ are still homogeneous, but their rates may reveal heterogeneity. The latter manifests itself as a jump or discontinuity across the surface delimiting the band, as illustrated in Fig. 2.

Because compatibility requires the velocity field $\mathbf{v}$ to remain continuous along the discontinuity surface, the jump of its gradient $\dot{\mathbf{F}}=\nabla \mathbf{v}$, by virtue of Maxwell's theorem, must take the form:

$$
\llbracket \dot{\mathbf{F}} \rrbracket=\dot{\mathbf{F}}^{b}-\dot{\mathbf{F}}=\dot{\mathcal{C}}^{0} \otimes \mathscr{N}^{0}
$$

where $\mathcal{N}^{0}$ is the unit vector normal to the band in the reference configuration. Vector $\dot{\boldsymbol{C}^{0}}=\llbracket \dot{\mathbf{F}} \rrbracket \cdot \mathscr{N}^{0}$ must be non-zero for effective bifurcation, and it defines the localization mode.

The second required condition is that of the stress equilibrium along the discontinuity surface:

$$
\dot{\mathbf{B}}^{b} \cdot \mathscr{N}^{0}=\dot{\mathbf{B}} \cdot \mathscr{N}^{0} \Leftrightarrow \llbracket \dot{\mathbf{B}} \rrbracket \cdot \mathscr{N}^{0}=\mathbf{0}
$$

Assume now that the constitutive law is written as

$$
\dot{\mathbf{B}}=\mathcal{L}: \dot{\mathbf{F}}
$$

This form will be derived later from the expression $\mathbf{N}=\mathbf{L}: \mathbf{G}$, involving the nominal stress $\mathbf{N}$ and Eulerian velocity gradient G, given in Section 2.2, Eq. (28). Combining Eqs. (36-38) yields

$$
\left\{\mathcal{L}: \dot{\mathcal{C}}^{0} \otimes \mathscr{N}^{0}\right\} \cdot \mathscr{N}^{0}=\left\{\left(\mathcal{L}-\mathcal{L}^{b}\right): \dot{\mathbf{F}}^{b}\right\} \cdot \mathscr{N}^{0}
$$

Considering that at the onset of bifurcation, the tangent modulus, which depends on the current state of stresses and internal variables, is continuous (i.e., $\llbracket \mathcal{L} \rrbracket=\mathcal{L}^{b}-\mathcal{L}=\mathbf{0}$ ), then one obtains

$$
\left\{\mathcal{L}: \dot{\boldsymbol{C}}^{0} \otimes \mathscr{N}^{0}\right\} \cdot \mathscr{N}^{0}=\mathbf{0}
$$

If we denote by $\mathcal{L}^{T}$ as the fourth-order tensor obtained from $\mathcal{L}$ by permutation of the two first indices $i$ and $j$ (i.e., $\mathcal{L}_{i j k l}^{T}=\mathcal{L}_{j k l}$ ), then condition (40) can be expressed in indicial notation as

$$
\left(\mathcal{N}_{i}^{0} \mathcal{L}_{i j k l}^{T} \mathcal{N}_{l}^{0}\right) \dot{\mathcal{C}}_{k}^{0}=0 \Leftrightarrow\left(\mathscr{N}^{0} \cdot \mathcal{L}^{T} \cdot \mathscr{N}^{0}\right) \cdot \dot{\mathcal{C}}^{0}=\mathbf{0}
$$

and a necessary condition for a non-trivial solution (i.e., non-zero jump, $\dot{\boldsymbol{e}}^{0} \neq \mathbf{0}$ ) is

$$
\operatorname{det}\left(\mathscr{N}^{0} \cdot \mathcal{L}^{T} \cdot \mathscr{N}^{0}\right)=0
$$

Condition (42) corresponds to Rice's bifurcation criterion, expressed relative to a fixed reference configuration, and it amounts to seeking the singularity of the acoustic tensor $\mathscr{Q}=\mathscr{N}^{0} \cdot \mathcal{L}^{T} \cdot \mathscr{N}^{0}$. 
The expression of modulus $\mathcal{L}^{T}$, or similarly $\mathcal{L}$, which has been left aside thus far, can be obtained from the relation $\dot{\mathbf{N}}=\mathbf{L}: \mathbf{G}$. The latter was expressed in the previous section relative to the current configuration; with respect to the reference configuration, the expression can be written as $\dot{\mathbf{N}}=J \mathbf{F}^{-1} \cdot \mathbf{L}: \mathbf{G}=J\left(\mathbf{F}^{-1} \cdot \mathbf{L} \cdot \mathbf{F}^{-1^{T}}\right): \dot{\mathbf{F}}$. Comparing this last expression with Eq. (38), which can be rewritten as $\dot{\mathbf{N}}=\mathcal{L}^{T}: \dot{\mathbf{F}}$, the relationship between the tangent moduli $\mathcal{L}^{T}$ and $\mathbf{L}$ can be deduced as

$$
\mathcal{L}^{T}=J \mathbf{F}^{-1} \cdot \mathbf{L} \cdot \mathbf{F}^{-1}
$$

It is sometimes more convenient to take the current state as reference configuration. For computational considerations, it may be easier to check the localization criterion on the current state, which requires the expression of the criterion relative to that configuration. To do this, the easiest way is to reconsider Eq. (42) taking the current state as reference (i.e., updated Lagrangian approach). In this case, $\mathbf{F}=\mathbf{1}$, which yields $\mathcal{L}^{T}=\mathbf{L}$ from (43), and the criterion becomes

$$
\operatorname{det}(\mathscr{N} \cdot \mathbf{L} \cdot \mathcal{N})=0
$$

In this last equation providing the Rice criterion with respect to the current configuration, $\mathcal{N}$ denotes the unit vector normal to the localization band in the current state. It is related to the normal in the undeformed configuration via Nanson's relation $\mathcal{N}=\frac{d S^{0}}{d S} J\left(\mathbf{F}^{-1}\right)^{T} \cdot \mathscr{N}^{0}=\frac{d S^{0}}{d S} J \mathcal{N}^{0} \cdot \mathbf{F}^{-1}$, in which $d S^{0}$ and $d S$ are the area elements in the reference and current configurations, respectively. Another straightforward way to derive Eq. (44) is by substituting in Eq. (42) the tangent modulus $\mathcal{L}^{T}$ and the normal $\mathscr{N}^{0}$ by their expressions from Eq. (43) and Nanson's relation, respectively.

\subsection{Strong ellipticity condition}

Another localization condition that was suggested and used by several authors is the loss of strong ellipticity (Bigoni and Hueckel, 1991; Neilsen and Schreyer, 1993). Because Rice's bifurcation corresponds to a loss of ellipticity, this strong ellipticity condition is expected to be more conservative than Rice's bifurcation exclusion. As discussed later, based on the analysis of eigenvalues of the tangent moduli that enter into play, the strong ellipticity condition is effectively more conservative than Rice's bifurcation exclusion. In other words, the singularity of the acoustic tensor cannot occur before the loss of strong ellipticity, and as a consequence, the limit strains predicted by Rice's criterion are always slightly higher than those determined by the loss of strong ellipticity (i.e., localization is slightly delayed with Rice's discontinuous bifurcation criterion).

A substantial amount of work has been conducted to investigate the localization predictions resulting from the condition of loss of strong ellipticity. Analytical formulas for the critical 
hardening moduli associated with localization have been sought mainly within the small-strain framework. For the associative plastic flow rule, we can cite the work of Bigoni and Hueckel (1990). For non-associative plasticity, the developments of Bigoni and Zaccaria (1992a,b) are notable. Finally, interesting analyses and analytical derivations of the critical hardening moduli associated with the condition of loss of strong ellipticity have been carried out when anisotropy only originates from the fourth-order elasticity tensor (Rizzi and Loret, 1997; Loret and Rizzi, 1997a) or when both elasticity and plasticity are anisotropic (Loret and Rizzi, 1997b).

Justification for the use of the condition of loss of strong ellipticity as a bifurcation criterion is closely related to the sufficient condition for uniqueness given by Hill (1958). This necessary condition for the loss of uniqueness was subsequently referred to as the general bifurcation criterion or more commonly as the loss of positiveness of the second-order work. Note that the satisfaction of this version of Hill's condition for uniqueness excludes the possibility of any type of bifurcation (diffuse or localized) and thus appears as a lower bound for all of the bifurcation criteria discussed in this study. It can be written locally as

$$
\dot{\mathbf{B}}: \dot{\mathbf{F}}>0
$$

The designation 'general bifurcation' for Hill's criterion, as opposed to the discontinuous bifurcation described by Rice, is explained by the fact that the loss of positiveness of the secondorder work, by violation of condition (45), mainly predicts diffuse bifurcation modes, while discontinuous (localized) modes are predicted in Rice's approach. Eq. (45) clearly shows that there is no restriction on the velocity gradient $\dot{\mathbf{F}}$ and thus the associated deformation mode. Thus, condition (45) will detect diffuse localization modes unless constraints related to the applied loading and prescribed boundary conditions prevent any diffuse bifurcation mode. When diffuse necking is excluded by applied loading or prescribed boundary conditions (see, e.g., Rice, 1976), Hill's criterion will primarily detect localized modes in the same manner as Rice's bifurcation criterion or the condition of loss of strong ellipticity. Using the elastic-plastic behavior law expressed by relation (38), Eq. (45) can be equivalently rewritten as

$$
\dot{\mathbf{F}}: \mathcal{L}: \dot{\mathbf{F}}>0, \quad \forall \dot{\mathbf{F}} \neq \mathbf{0}
$$

which amounts to the condition of positive-definiteness of the elastic-plastic constitutive modulus $\mathcal{L}$; the latter condition being classically equivalent to the condition of positive eigenvalues for the symmetric part $\mathcal{L}^{\mathrm{s}}$ of $\mathcal{L}$, defined as $\mathcal{L}^{\mathrm{s}}{ }_{i j k l}=\left(\mathcal{L}_{i j k l}+\mathcal{L}_{k l i j}\right) / 2$.

If we restrict the condition of positive-definiteness (46) to the localized deformation modes (i.e., those satisfying the compatibility condition $\dot{\mathbf{F}}=\mathscr{M}^{0} \otimes \mathscr{N}^{0}$ ), then the strong ellipticity condition is recovered as follows: 


$$
\mathscr{M}^{0} \otimes \mathscr{N}^{0}: \mathcal{L}: \mathscr{M}^{0} \otimes \mathcal{N}^{0}>0, \quad \forall \mathscr{M}^{0}, \mathscr{N}^{0} \neq \mathbf{0}
$$

It is then clear that the general bifurcation exclusion (Eq. (46)) is more conservative than the strong ellipticity condition (Eq. (47)) because in the former, the condition of positive-definiteness for the constitutive tensor is required on a larger vector space. This provides a mathematical interpretation of what is commonly observed experimentally, namely that diffuse necking occurs prior to localized necking. Other interpretations that present localized and diffuse modes in terms of bifurcations associated with compatible and incompatible deformation, respectively, can also be found in the literature (Neilsen and Schreyer, 1993). It has been shown that a bifurcation mode associated with incompatible deformation may only appear in a zone of measure zero of the solid (i.e., at the boundary), while a localized mode occurs in the interior of the solid.

It is also important to compare the strong ellipticity condition with Rice's criterion for exclusion of discontinuous bifurcation. To this end, Eq. (47) can be conveniently rewritten using modulus $\mathcal{L}^{T}$, which was previously defined as $\mathcal{L}_{i j k l}^{T}=\mathcal{L}_{j i k l} ;$ one obtains

$$
\mathscr{M}^{0} \cdot\left(\mathscr{N}^{0} \cdot \mathcal{L}^{T} \cdot \mathscr{N}^{0}\right) \cdot \mathscr{M}^{0}>0, \quad \forall \mathscr{M}^{0}, \mathscr{N}^{0} \neq \mathbf{0}
$$

The acoustic tensor, denoted $\mathscr{Q}=\mathscr{N}^{0} \cdot \mathcal{L}^{T} \cdot \mathcal{N}^{0}$, as discussed previously, is then revealed by Eq. (48). In other words, the strong ellipticity condition is equivalent to the positive-definiteness of the acoustic tensor (or more precisely of its symmetric part), which equivalently amounts to verifying the positiveness of the eigenvalues of the second-order tensor $\mathfrak{Q}^{s}$, which is defined as $\mathscr{Q}^{s}=\left(\mathscr{Q}+\mathscr{Q}^{T}\right) / 2$. Because it is mathematically shown that the real parts of the eigenvalues for a given tensor are bounded by the smallest and largest eigenvalues of its symmetric part, it is clear that the strong ellipticity condition is more conservative than Rice's criterion for exclusion of discontinuous bifurcation. In other words, the singularity of the acoustic tensor cannot occur before the loss of strong ellipticity, and thus, localization predictions based on the loss of strong ellipticity are generally more conservative than those associated with Rice's criterion (i.e., loss of strong ellipticity predicts earlier localization as compared to Rice's bifurcation criterion).

However, it is interesting to note that in the framework of small strains and associative plasticity, the tangent modulus that enters the expression of the acoustic tensor has the major symmetry. This implies that the acoustic tensor is symmetric and thus only has real eigenvalues. In such conditions, the strong ellipticity condition (positive-definiteness of the symmetric part of the acoustic tensor) and Rice's bifurcation exclusion (non-singularity of the acoustic tensor) are equivalent.

In the same manner as before, the above derivations for the general bifurcation criterion and strong ellipticity condition have been expressed relative to the initial undeformed configuration. 
Their expressions with respect to the current deformed configuration are easily obtained using the updated Lagrangian concept. In the latter approach, the strong ellipticity condition can be written as

$$
\mathcal{M} \cdot(\mathscr{N} \cdot \mathbf{L} \cdot \mathscr{N}) \cdot \mathcal{M}>0, \quad \forall \mathscr{M}, \mathscr{N} \neq \mathbf{0}
$$

where $\mathbf{L}$ is the tangent modulus relating the nominal stress rate $\dot{\mathbf{N}}$ to the velocity gradient $\mathbf{G}$ (see Eqs. (15) and (28)). We thus recognize the condition of positive-definiteness of the symmetric part of the acoustic tensor, which can be compared in the same manner to the condition of singularity of the acoustic tensor (see Eq. (44)).

Summarizing the comparison of the three above-discussed criteria, it has been shown that the general bifurcation criterion (Eqs. (45) or (46)), which primarily predicts diffuse modes, is the most conservative of the three criteria, and the second most conservative criterion is associated with the strong ellipticity condition (Eqs. (47), (48) or (49)), which predicts localized modes. Finally, the least conservative criterion appears to be Rice's bifurcation criterion, which is associated with the loss of ellipticity and predicts shear band localization (see Eqs. (42) or (44)). Considering that a more conservative criterion predicts earlier plastic instability, the critical strains predicted by Rice's bifurcation criterion represent an upper bound for the three above-discussed criteria. Because it is now widely recognized that the critical strains limiting sheet metal formability are those associated with plastic strain localization, our focus in the following sections will be confined to the two localization criteria given by the loss of strong ellipticity and Rice's discontinuous bifurcation.

\section{Prediction of ductility limits for single crystals and polycrystals}

In this section, the previous developments will be applied to the investigation of ductility limits for single crystals and then polycrystalline aggregates. As reported by Iwakuma and Nemat-Nasser (1984), during plastic flow in polycrystals undergoing large deformations, individual grains may reach various geometries and orientations resulting in the local loss of stability within some grains well before the attainment of the overall instability condition characterized by strain localization. Although the instability condition may be reached in some grains, the stresses imposed by other neighboring grains maintain the overall response stable up to a higher level in the loading process. During this process, an increasing number of grains will become locally unstable until the overall localization of the plastic flow manifests itself.

Because the main objective of this study is to predict the loss of strong ellipticity and loss of ellipticity for the overall response with maximum accuracy, considerable attention has been paid to the most important softening effects within the proposed modeling. Several possible sources of softening are effectively included in the constitutive modeling. For instance, considering the crystallographic lattice rotation for each grain in the course of the macroscopic loading applied to 
the polycrystal may contribute to promoting overall instability. Moreover, the adopted hardening, which accounts for dislocation annihilation, introduces another softening effect that should be favorable to localization. Likewise, the possibility of multi-slip, which is a characteristic of crystal plasticity in relation to the yield surface vertex formation, is another key parameter in the prediction of localization. The convective terms of stresses, which are induced by the large-strain framework, may also have a non-negligible effect. Finally, textural softening and induced anisotropy (both crystallographic and morphological) are also taken into account and should play an important role in the analysis (see, e.g., Zhao et al., 2008; Kobayashi, 2010; Shanthraj and Zikry, 2012).

Note that the application of Rice's localization analysis to the prediction of forming limit diagrams for polycrystalline steels has already been carried out in some of our earlier works (see, e.g., Franz et al., 2009a,b). The aim of this study is to disclose the impact of microstructural parameters on the ductility limit of such materials. Because the developed predictive tool is partly intended to help design steels with improved ductility properties, the analysis of the effect of certain physical material parameters may prove to be particularly informative.

\subsection{Prediction of strain localization for single crystals}

In this section, we reconsider the single crystal constitutive equations developed in Section 2 by coupling them with the bifurcation analysis detailed in Section 3. Although the prediction tool developed here is primarily intended for polycrystalline materials, its preliminary application to the localization analysis of single crystals aims to verify the expected general trends and validate the approach with regard to the main expected effects. Thus, we start by investigating the effect of certain physical mechanisms on the ductility limit of single crystals with parameters $\tau_{0}^{g}, y_{c}$, and $g_{0}$, which denote, the initial critical shear stress, critical annihilation distance of dislocations, and parameter related to the mean free path of dislocations, respectively. Because some effects may have been inhibited or considerably reduced through the scale-transition scheme, analyzing them before and after this averaging process was perceived to be important. Moreover, to confirm the general trends regarding the effect of these physical parameters, two different scale-transition schemes will be applied and compared, namely the self-consistent approach and the full-constraint Taylor model. To begin with, the condition of singularity of the acoustic tensor and that relating to the loss of positive-definiteness of its symmetric part are analyzed for the single crystal. The acoustic tensor associated with the single crystal is written as

$$
\mathscr{Q}=\mathscr{N} \cdot \mathbf{l} \cdot \mathscr{N}
$$

where $\mathbf{l}$ is the single crystal tangent modulus given in Eqs. (15) and (23), relating the nominal stress rate $\dot{\mathbf{n}}$ to the velocity gradient $\mathbf{g}$. 
The theoretical application of Rice's bifurcation criterion and the condition of loss of strong ellipticity should be undertaken in the following manner: at every point of a given loading path, one checks the condition of the singularity of the acoustic tensor and the condition of the loss of positive-definiteness of the symmetric part of the acoustic tensor for all possible unit normal vectors. In practice, this normal $\mathcal{N}$ is defined using two angles $\theta_{1}$ and $\theta_{2}$ in three-dimensional space, both angles are discretized here with an angular increment size of $1^{\circ}$, and a systematic search is performed over all of the corresponding orientations in the discretized space. When one or the other of these criteria is satisfied at a given point of the considered loading path, the corresponding state of deformation is recorded, which provides the limit strains, while the associated normal vector provides the three-dimensional orientation of the localization band.

Note that in these theoretical localization criteria, diffuse necking, which may occur in experiments before localized necking, is not taken into account. Accordingly, the boundary value problem is set in such a way that diffuse necking does not arise. In other words, the conditions of homogeneous deformation until localization are imposed (i.e., no structural/geometric effects are considered). In the literature (see, e.g., Saje et al., 1982), this can be obtained by prescribing allaround displacement boundary conditions, so that necking modes are ruled out. In our approach, which is commonly followed in FLD analyses, we only consider a single material point to which various loading paths are applied in order to reproduce the traditional loading paths that are required to determine FLDs. This also amounts to considering a single finite element, with a single integration point, which guaranties a uniform state of deformation until localization is detected. The motivation behind these conditions of uniform pre-localization state is to allow consistency with the bifurcation criteria, in which bifurcation is assumed to initiate from a uniform deformation state.

For a single crystal with the initial crystallographic orientation (ND)[RD] $=(5-12-10)[201]$, as given in Table 1 and illustrated in Fig. 3, and with the material parameters listed in Table 1, in which parameter $D$ is taken to be equal to the smallest dimension of the sample, uniaxial tensile loading paths will be applied in different directions with respect to the rolling direction (RD), and the effect of certain physical parameters on the ductility limit will be investigated. It is worth noting that the complete validation of the proposed single crystal constitutive modeling was achieved through a comparison with experimental results for linear and sequential loading paths for single crystals with various initial crystallographic orientations (Lorrain, 2005). However, the current investigation of the impact of certain physical parameters on the ductility limit of single crystals should be viewed as a qualitative analysis, as no comparison data are available for such an analysis.

In the process of searching for the minimal value of the determinant of the acoustic tensor, it was observed that this function had abrupt variations with respect to the normal direction and revealed several local minima. The same observations apply to the minimal eigenvalue of the symmetric part 
of the acoustic tensor. Thus, in this minimization process, we have chosen to discretize the entire space of orientations for the normal to the localization band, which, although time consuming, appears to be a secure way to determine the global minimum. For single crystals with special initial orientations, the well-known results regarding the orientation of the localization band in the uniaxial tensile test are recovered (Lorrain, 2005). However, the orientation of the normal defining this localization band as well as the corresponding limit strains are found to be strongly dependent on the initial orientation of the single crystal, whose behavior is highly anisotropic due to its initial crystallographic orientation and its evolution.

The effect of the initial critical shear stress is illustrated in Figs. 4 and 5. In this preliminary analysis, all of the parameters in Table 1 are held constant except the initial critical shear stress $\tau_{0}^{g}$, which is varied to investigate its impact on localization. At the top of Figs. 4 and 5, the stress-strain responses for uniaxial tensile tests performed parallel to the rolling direction are given for different values of the initial critical shear stress. The end of each stress-strain curve (marked by a cross) corresponds to the limit strain associated with localization as predicted by one or the other criterion. At the bottom of Fig. 4, the minimal value of the cubic root of the determinant of the acoustic tensor over all of the orientations for the normal to the localization band is plotted as a function of the strain for different values of the initial critical shear stress. Likewise, at the bottom of Fig. 5, the minimal value of the smallest eigenvalue of the symmetric part of the acoustic tensor over all of the orientations for the normal to the localization band is plotted as a function of the strain for different values of the initial critical shear stress. One can observe that the minimal determinant initially exhibits rapid variation at the elastic-plastic transition and subsequently when localization is approached (i.e., when the value of the determinant tends to zero). The same observation applies to the minimal eigenvalue of the symmetric part of the acoustic tensor. Larger values for the initial critical shear stress (elastic limit) result in reduced ductility for the material. These results correspond well with those reported by Luft (1991), who carried out uniaxial tensile experiments on single crystals of molybdenum with the same physics for plastic flow as steels.

Another observation is that the limit strains given by the bifurcation analysis (see Fig. 4) are very close to the limit strains obtained from the criterion of loss of strong ellipticity (see Fig. 5). This result, which will be further interpreted later, has been observed in all of the parametric studies conducted in this section for single crystals with varying parameters. Consequently, for the sake of conciseness, only one pair of results yielded by the two criteria are provided here (see Figs. 4 and 5), while in the remaining of the analysis, the results correspond to those obtained with the bifurcation criterion. Note, however, that although the resulting limit strains for the two criteria are nearly identical, the evolution of the determinant of the acoustic tensor until the loss of ellipticity 
differs significantly from the evolution of the smallest eigenvalue of its symmetric part until the loss of strong ellipticity (see, e.g., the corresponding evolutions in Figs. 4 and 5).

Fig. 6 illustrates the effect of the critical annihilation distance of dislocations $y_{c}$ on the ductility limit of single crystals. Materials with low values of critical annihilation distance can store more dislocations, thus improving their ductility. Another way to interpret this effect is to examine the evolution equations of dislocation densities (Eqs. (25) and (26) in Section 2.1). The mathematical structure of these ordinary differential equations suggests that they can be integrated in the form of a saturating response when the annihilation term $y_{c}$ is present. The latter parameter quantifies the importance of annihilation and acts as a saturation rate in the same manner as in the Voce isotropic hardening law. Thus, larger values of this parameter lead to faster saturation, which has a destabilizing effect. In other words, this softening effect, due to dislocation annihilation, contributes to the overall softening of the material, thus promoting early plastic strain localization.

Finally, the effect of a last parameter, $g_{0}$, which is related to the mean free path of the dislocations, on the ductility limit of single crystals is studied. Fig. 7 reflects this effect and shows that larger values of this parameter imply larger values for the mean free path of the dislocations, thus reducing the dislocation storage in the material and hence its work-hardening capability, which ultimately leads to decreased ductility. Examination of the evolution equations of the dislocation densities (Eqs. (25) and (26) in Section 2.1) allows us to interpret this effect again. Parameter $g_{0}$ affects the first term of the right-hand side of Eq. (25), which corresponds to the dislocation storage term, whose effect is the opposite of that of the dislocation annihilation term. Thus, an increase in parameter $g_{0}$ will reduce the dislocation storage term, thus promoting early localization. These results indicate that this parameter is a limiting factor for ductility, as shown in Fig. 7.

To investigate the dependence of strain localization on the single crystal orientation, the analysis is repeated with different orientations for the tensile direction. The impact on the predicted limit strains of varying the loading direction is analyzed for two other tensile axis orientations: at $45^{\circ}$ and $90^{\circ}$ with respect to the rolling direction. Is has been found that the effects of the previously investigated physical parameters are consistently reproduced; however, for both loading directions, the predicted limit strains have increased. The results are shown in Figs. 8 to 10 for the loading direction corresponding to the largest increase in the limit strains (i.e., parallel to the transverse direction (TD)), while the $45^{\circ}$ direction (not shown here) corresponds to the intermediate values for the predicted limit strains. Note that for the tensile test performed parallel to the transverse direction, the evolution of the minimal determinant of the acoustic tensor features an intermediate increase around a tensile strain value of 0.05 before decreasing again towards zero (see Figs. 8 to 10). This has also been observed for the loading direction at $45^{\circ}$ with respect to RD, yet with a less 
pronounced peak, which may be attributable to the rotation of the single crystal towards a preferred more stable orientation, before experiencing strain localization at a higher strain level.

As already observed above, in all of these investigations reported in Figs. 4 to 10, the limit strains obtained by Rice's criterion (i.e., singularity of the acoustic tensor) are found to be close to those yielded by the loss of strong ellipticity (i.e., the loss of positive-definiteness of the symmetric part of the acoustic tensor). This result is understandable when examining the algebraic structure of the single crystal tangent modulus (see, e.g., Eq. (23)), revealing that although this tangent modulus has none of the minor or major symmetries, the convective stress components responsible for this loss of symmetry are small compared to the elasticity tensor. Under these conditions, the resulting acoustic tensor and its symmetric part, although distinct, will only differ by components that are small compared to the principal part of the acoustic tensor. Whenever this situation is encountered, the two criteria, although mathematically different, would provide eigenvalues of the same order of magnitude, thus resulting in comparable limit strains.

Note also that other investigations involving various single crystals with different initial orientations have confirmed that the same qualitative effects concerning the above-discussed physical parameters are consistently demonstrated (Lorrain, 2005). These aspects, which are closely related to microstructure, could be taken advantage of in the design of new steels and particularly in the optimization of their properties in terms of formability.

\subsection{Application to the prediction of the ductility limits of polycrystals}

We now consider the case of a polycrystalline material, and we will similarly investigate the effect of the same physical and microstructural parameters on the ductility limit. As described previously, an initial set of parameters, which is given in Table 2, is selected, and then one parameter is varied at a time to investigate each parameter respective impact on ductility. For comparison purposes, the two scale-transition schemes discussed in Section 2.2, namely the selfconsistent averaging rule and the full-constraint Taylor model, will be successively applied.

For this qualitative study, an initial texture defined by 1,000 crystallographic orientations, as illustrated by the associated pole figures given in Fig. (11), is selected to reproduce the ferritic single-phase steel, denoted IF-Ti, which was previously studied in the validation process of the proposed constitutive modeling. Accordingly, the initial set of parameters in Table 2, before any variation, corresponds to the material parameters identified for the IF-Ti single-phase steel, which will be studied later and include a comparison of its stress-strain responses with experimental tests. Applying Rice's criterion (i.e., singularity of the acoustic tensor associated with the effective tangent modulus of the polycrystal) and the condition of loss of strong ellipticity (i.e., the loss of positive-definiteness of the symmetric part of the acoustic tensor) reveals the same observations as 
in the case of single crystals. The determinant of the acoustic tensor and the smallest eigenvalue of its symmetric part show strong variations with the normal orientation, although this result is less marked than in the case of single crystals, most likely due to the averaging effect, and reveal several local minima. Once again, this behavior does not allow the use of conventional efficient minimization procedures, and the recourse to the systematic discretization of the orientation space for the normal to the localization band appears to be a secure way to detect the global minimum. Another noteworthy observation is that, as found in the case of single crystals, the limit strains predicted by the two criteria are close due to the small effect of the stress components responsible for the loss of symmetry in the single crystal tangent modulus and, by way of the averaging rule, in the polycrystal effective tangent modulus.

Figs. 12 to 15 illustrate the effects of parameters $\tau_{0}^{g}, y_{c}$, and $g_{0}$ on the ductility limit of the polycrystal when applying the self-consistent scale-transition scheme, while Figs. 16 and 17 reflect the same parametric study using the full-constraint Taylor model. The same effects are found for the uniaxial tensile test and the plane strain tensile test, both having been performed parallel to the rolling direction. Consequently, the results will be shown for the plane strain tensile test because the associated limit strain corresponds to the lowest point of the FLD, which gives a good indication of the overall forming limit and thus the material ductility. It is worth noting that in all of the localization analyses reported in Figs. 4 to 10 and Figs. 12 to 17, the bifurcation instability is predicted in the positive hardening regime (i.e., before the peak in the true stress-strain curve), in contrast to phenomenological flow theories with associative plasticity and smooth yield surface. This is made possible here thanks to the yield surface vertex structure inherent in crystal plasticity, which arises from the discrete nature of crystalline slip.

The same trends observed for single crystals are found for polycrystals. An increase in the initial critical shear stress (elasticity limit) results in decreased ductility. Figs. 12 and 13 reveal this effect with the self-consistent scheme, while Fig. 16 confirms the same trend using the full-constraint Taylor model. Note that in the latter case of the Taylor model, the predicted limit strains are significantly higher when compared to those predicted by the self-consistent scheme. Also, as obseryed for single crystals, the limit strains predicted by bifurcation analysis in Fig. 12 are very close to those given by the criterion of loss of strong ellipticity in Fig. 13. The top of Figs. 12 and 13 are very similar, while their bottoms display different evolutions of the localization indicators. The same applies to Fig. 16, in which Taylor's averaging rule has been adopted. Because the two localization criteria provide almost the same limit strains, only the results of the Rice bifurcation analysis will be shown in the remaining of the parametric study.

For the effect of the critical annihilation distance of dislocations, similar to single crystals, Fig. 14, corresponding to the self-consistent scale-transition scheme, and Fig. 17 (top), associated with 
the Taylor model, reflect the same trends. Analogously, larger values for the critical annihilation distance of dislocations $y_{c}$ tend to reduce the overall ductility of the polycrystalline aggregate. A noteworthy observation is that, once again, the limit strains predicted by the Taylor model are much larger than those provided by the self-consistent scheme.

Finally, the effect of parameter $g_{0}$, which is related to the mean free path of the dislocations, on the ductility limit of polycrystals is illustrated in Fig. 15, using the self-consistent scheme, and in Fig. 17 (bottom), with the Taylor model. These findings suggest conclusions quite similar to those for single crystals, namely an increase in the mean free path of the dislocations reduces the overall ductility of the polycrystalline aggregate. Consistent with the previous analyses, the Taylor model predicts limit strain levels sensibly higher compared with those given by the self-consistent scheme.

Another observation from the above parametric studies is that the strain spacing between consecutive curves at the point of localization seems to be more regular in the case of single crystals (see Figs. 4 to 10) than for polycrystals (see Figs. 12 to 17). This is likely attributable to the fact that only one single local tangent modulus is involved in the acoustic tensor associated with a single crystal, while some thousand microscopic tangent moduli enter into play in the case of polycrystalline aggregates. The latter tangent moduli may differ significantly from grain to grain, exhibiting high contrast in their components due to texture evolution, which results in a rather complex evolution for the minimal value of the determinant of the polycrystal acoustic tensor over all orientations for the normal to the localization band. It is also found that this evolution is less regular in the case of the Taylor model than for the self-consistent scheme, most likely due to the corresponding averaging rule (i.e., simple volume average of the microscopic tangent moduli).

To summarize, it is shown that the localization analyses conducted for single crystals in the previous section can be extended in the same way to polycrystals. It follows that the investigation of the impact of physical material parameters yields consistent results for polycrystalline aggregates because the same trends as those found for single crystals are revealed. Note that although the same trends are reflected by the Taylor model, the corresponding limit strain levels appear to be unusually high as compared to those yielded by the self-consistent scale-transition scheme. Moreover, this methodology and the associated localization criteria can be applied to determine FLDs for polycrystalline steels. It has been shown in previous investigations that the FLDs resulting from application of the self-consistent scheme for single-phase ferritic or dual-phase ferriticmartensitic steels correspond well with the results available in the literature (see Franz et al., 2009a,b). To illustrate the similarity of the results, we will show an example of an FLD determined for a single-phase steel (IF-Ti) compared to the reference results.

It is important to note that before evaluating the proposed modeling in terms of its capability to predict FLDs, we have first validated the polycrystalline constitutive modeling through comparison 
with experimental tests for linear and sequential loading paths. For the numerical simulations, various calculations have been performed by varying the number of grains, and an initial texture composed of 1,000 crystallographic orientations yielded by the orientation distribution function has been ultimately selected, based on a compromise in terms of accuracy and calculation time. The four material parameters must be identified; to this end, two mechanical tests have been systematically used for each material. Generally, a uniaxial tensile test and reverse (Bauschinger) shear test are used to identify parameters $y_{c}$ and $g_{0}$. The average grain size $D$ is obtained from the steel micrography, while the initial critical shear stress, which is assumed to be the same for all slip systems, is determined based on the elastic limit (approximately half of the elastic limit in the uniaxial tensile test). The latter is justified by the fact that the overall material response corresponds to an averaging rule over a large number of grains with different orientations, and accordingly, it is reasonable to assume that the loading orientation has little effect on the elastic limit.

For the selected single-phase steel, denoted as IF-Ti, the identified parameters are reported in Table 2. Note that in this validation process of the polycrystalline constitutive modeling, only one or two mechanical tests have been used for identification, whereas the remaining tests provided by ArcelorMittal have been used for validation purposes. Fig. 18 shows a comparison example between the simulation results and experimental tests. By convention, for uniaxial and plane strain tensile tests, the Cauchy stress component $\sigma_{11}$ is plotted as a function of the logarithmic strain component $\varepsilon_{11}$; for simple and reverse (Bauschinger) shear tests, the Cauchy stress component $\sigma_{12}$ is expressed as a function of the shear strain component $\gamma=2 \varepsilon_{12}$; for cross (orthogonal) tests, the Cauchy stress component $\sigma_{11}$ is plotted as a function of the logarithmic strain component $\varepsilon_{11}$, during the prestrain (i.e., the plane strain tensile test), and then the Cauchy stress component $\sigma_{12}$ is represented as a function of the shear strain component $\gamma=2 \varepsilon_{12}$, augmented by the amount of prestrain, for the subsequent loading path (i.e., the simple shear test).

Overall, the obtained results correspond reasonably well with the experimental findings, considering the wide range of steels tested, which have different microstructures and mechanical characteristics (Franz, 2008; Franz et al., 2009a,b). The discrepancies observed during the reverse (Bauschinger) shear tests and cross (orthogonal) tests are attributable to the spatial rearrangement of the dislocation cells that are not properly taken into account in the proposed constitutive modeling. Experimental evidence for the formation of such intragranular dislocation substructures has been shown for ferritic steels (Nesterova et al., 2001). These intragranular microstructures, which are produced along the first strain path, will disintegrate in favor of the creation of a new dislocation substructure, which depends on the second loading path. It can also be observed that these transient effects (e.g., work-hardening stagnation for the Bauschinger shear tests and the softening regime for 
the cross tests), which occur after the strain-path changes, are increasingly pronounced as the amount of prestrain increases. In this case, the prestrain-induced intragranular microstructure will be more persistent and difficult to disintegrate during subsequent paths. To account for these effects, advanced constitutive models have been recently developed to reproduce these softening/hardening transient phenomena, which are characteristic of strain-path changes. Examples include the Teodosiu-Hu model (Teodosiu and $\mathrm{Hu}, 1998$ ), which although phenomenological, is based on dislocation microstructures, and the Peeters model (Peeters, 2002), which is micromechanical but appears to be restricted to small strains.

Examples in terms of FLD results are presented here for the selected IF-Ti steel compared to reference linear FLDs. Because Rice's localization criterion and the condition of loss of strong ellipticity predict almost identical limit strains, only the FLDs corresponding to Rice's bifurcation criterion are shown. To illustrate the effect of strain-path changes, sequential two-stage FLDs are also determined. Fig. 19 shows FLDs with linear strain paths obtained with the proposed polycrystal self-consistent modeling compared to available reference FLDs. The latter, which is referred to as the ArcelorMittal FLD model, is obtained based on the approach proposed by Cayssials (Cayssials, 1998; Cayssials and Lemoine, 2005). This approach has been validated by ArcelorMittal over a wide range of sheet metal grades with systematic comparison to experimental FLDs. Despite the good prediction capability of ArcelorMittal's FLD model, its limitation lies primarily in its restriction to linear strain-path FLDs together with its phenomenological basis, which cannot take microstructural effects into account. This deficiency is the main motivation behind the development of the proposed modeling, which seeks to provide a predictive tool that can be used in the design of new grades of steels. Recall that the experimental measurement of limit strains is a difficult, time-consuming, and expensive task.

Inspection of Fig. 19 reveals some differences between the two FLDs. It should first be noted that, while the proposed FLD model makes use of bifurcation theory, the ArcelorMittal FLD model is based on a quite different approach. In the latter, the effect of diffuse necking, which inherently develops in sheet metals before localization, is taken into account in an approximate phenomenological way, whereas in the former, localization bifurcation stems from an assumed uniform deformation state. The fact that consideration of diffuse necking in the analysis is known to reduce the predicted limit strains may explain the overall lower level of ArcelorMittal's FLD. The two FLDs are closer in the neighborhood of the plane strain tension point, which may be explained by the fact that the amount of deformation taking place from diffuse necking to localization is much smaller for this loading path. Other sources that are likely to explain the observed differences include: the structural effects that are unavoidably present in the experimental determination of FLDs and not considered in the bifurcation approach, the limitations in the material parameter 
identification procedure and the associated issue of the proper stress-strain curve extrapolation at large strains, the regularization method adopted for slip system selection, which has been shown to result in relatively stiffer overall moduli, which in turn would increase the predicted limit strain levels. In view of the above issues, the comparison between theoretical and experimental FLDs should only be considered qualitatively (i.e., with respect to the overall strain levels predicted at localization). In the same way, we would suggest a more intrinsic use of the ductility limits afforded by bifurcation theory, which is to compare and classify materials in terms of their formability, and to serve in the design process of new grades of steel having improved ductility properties.

Another observation from Fig. 19 concerns the non-smoothness of the path followed by the predicted FLD points. In contrast to the smooth nature of FLDs obtained with phenomenological constitutive models, FLDs determined either experimentally (see, e.g., Knockaert et al., 2002; Kuwabara, 2007; Signorelli et al., 2012) or using crystal plasticity models (see, e.g., Barlat, 1987; Wu et al., 1997; Yoshida et al., 2007) generally exhibit some scatter. This is very likely attributable to the texture development and its evolution in crystal plasticity descriptions. In such models, the homogeneous deformation imposed macroscopically will result in deformation states quite different from grain to grain, leading to a rather complex texture evolution. In other words, micromechanicsbased descriptions should be viewed as macroscopically homogeneous, while heterogeneous at the microscale. Because such descriptions are more representative of the actual material behavior, this common feature may contribute to justify the non-smoothness of FLDs obtained either from crystal plasticity modeling or from experimental measurement. In Barlat (1987), where the M-K approach was used with yield surfaces obtained by crystal plasticity calculations following the TaylorBishop-Hill procedure, the observed scatter was explained by the fact that the resulting yield loci are smooth for the first order but not for the second order. Because the M-K method involves derivatives of these yield loci, this leads to non-smooth predicted FLDs. In crystal plasticity modeling, sharp vertices form on the current yield surface thus reducing its smoothness, which in turn affects the smoothness of the resulting tangent moduli. The latter are the key elements in the localization criteria (see, e.g., the construction of the acoustic tensor), and therefore responsible, to some extent, for the final form of the predicted FLDs and the degree of their smoothness.

A noteworthy discussion is also related to the difference in the limit strains predicted by the two averaging schemes (i.e., the self-consistent and Taylor's model). As revealed from the parametric study corresponding to the plane strain tensile test, the limit strains predicted by the full-constraint Taylor model are found to be much larger (see Figs. 16 and 17 compared to Figs. 12 to 15). This has also been observed for other loading paths and, notably, in the balanced biaxial tensile test where the Taylor predicted limit strains seem to be particularly overestimated. Recent investigations using rate-sensitive crystal plasticity models in conjunction with the M-K approach also suggested 
significant discrepancies depending on the adopted homogenization scheme (see, e.g., Signorelli et al., 2012). Several causes are likely to explain such observed differences. For example, the selfconsistent model, which accounts for grain interaction effects and local non-uniform deformations that occur in real polycrystals, has been shown to estimate a softer overall response for rateindependent polycrystals as compared to the full-constraint Taylor model (Hutchinson, 1970; Takahashi, 1988; Harren and Asaro, 1989). The stiffer overall response of the Taylor model (see Figs. 16 and 17 as compared to Figs. 12 to 15), which has been often considered as an upper bound for the stresses, may contribute to explain the associated higher predicted limit strains. Recent reexamination of the Taylor model (Tadano et al., 2012) also revealed that its predictions tend to underestimate the lattice rotations. Because the latter play a major role in inducing textural (geometrical) softening that is favorable to localization, this may bring some support to the aboveobserved differences. While one effect, such as texture development, could be the dominant cause, the combined contribution of different interacting effects, as discussed above, seems to be a more plausible explanation of these results.

Finally, to emphasize the impact of strain-path changes, which are inherent to most sheet metal forming processes, sequential FLDs obtained after prestrain consisting of either uniaxial tension or balanced biaxial tension are given in Fig. 20 for the IF-Ti single-phase steel. These results are qualitative, as no reference sequential FLDs are available, and ArcelorMittal's FLD model is not designed to predict formability when considering two-stage or more complex loading paths. Nonetheless, the available literature results for other materials reveal that FLDs obtained after prestrain tend to shift along the prestrain-path direction (Haddad, 1997; Stoughton and Zhu, 2004; Haddag et al., 2009). For instance, for uniaxial tensile prestrain, the FLD is shifted to the top and left, whereas the FLD is shifted to the bottom and right for balanced biaxial tensile prestrain. These qualitative results are consistently reproduced by the current modeling (see Fig. 20).

\section{Conclusions}

As described previously, the main objective of the proposed methodology is to establish a link between microstructure-related parameters and material ductility. This effort is part of a larger project in which a general multi-purpose predictive tool is intended to be advantageously used in the design of new and advanced steels with improved formability and in-use properties. The starting point of this approach is an advanced constitutive framework for single crystals, which is applicable in large-strain elasto-plasticity. Throughout this constitutive modeling, substantial effort has been devoted to the consideration of most key physical mechanisms that are relevant at the microscale, such as creation, storage, and annihilation of dislocations. It is believed that the accurate description 
of these microstructural mechanisms would play a crucial role in the prediction of plastic strain localization and the corresponding formability limits. In the same manner, the overall response of polycrystalline aggregates is derived based on the self-consistent scale-transition scheme, with the same attempt to account for the essential microstructural features and deformation mechanisms (e.g., initial and induced textures, dislocation density evolution, and softening mechanisms).

The resulting micromechanical constitutive modeling, both for single crystals and polycrystals, is then coupled with two strain localization criteria: Rice's bifurcation criterion, which is based on the loss of ellipticity of the associated boundary value problem, and the condition of loss of strong ellipticity. Mathematical consideration of these criteria, based on the analysis of the associated eigenvalues, allowed us to classify them in terms of conservativeness. More specifically, it has been shown that Rice's bifurcation criterion is less conservative, in the general case, than the condition of loss of strong ellipticity, thus leading to a higher level in terms of the predicted limit strains. However, it is shown that the two criteria coincide in the framework of small strains and associative plasticity. Likewise, in other specific cases, the two criteria may result in comparable predictions of the limit strains. This is the case when the structure of the relevant tangent moduli consists of a main part, which is identical and possesses all minor and major symmetries, and an additional part of stress components that is small compared to the main part.

Application of the proposed coupling to the prediction of the ductility limit for single crystals provided consistent results when compared to the predictions for polycrystals. The effect of physical microstructural parameters on material ductility has been systematically analyzed, with an attempt to provide some physical or mathematical interpretation. Because the convective stress components responsible for the loss of symmetry of the tangent modulus and thus the loss of symmetry of the acoustic tensor are small compared to the elasticity tensor, the predictions of the two criteria are found to be comparable. This result also provides some justification for the use of Rice's bifurcation theory as a strain localization criterion, which is also designated as plastic instability in the context of sheet metal formability. The predictive capability of the proposed approach has been shown through the determination of forming limit diagrams. Examples of FLDs for IF-Ti single-phase steels compared to reference results revealed consistent results. The simulations using the self-consistent scheme have been systematically compared to those of the Taylor model, and the latter have been found to predict higher limit strains, especially in balanced biaxial tension where the formability level seems to be particularly overestimated. The effect of strain-path changes has also been investigated by analyzing the impact of prestrain on the FLDs.

It is worth mentioning that this micromechanics-based methodology is related to the vertex effect induced by crystal plasticity, which was pointed out earlier by Hill (1967). This vertex formation on the current points of the yield surface is a key factor in flow localization analysis based on bifurcation 
theory or the loss of ellipticity, thanks to the associated reduction in the instantaneous shear moduli (see, e.g., Hutchinson, 1970; Yoshida et al., 2009). Such an analysis using phenomenological flow theories with associative plasticity and smooth yield surface requires softening effects (Needleman and Tvergaard, 1992), which could be introduced by coupling with damage. Another possibility for triggering bifurcation in the hardening regime within phenomenological modeling is the use of the deformation theory of plasticity rather than flow theory (see, e.g., Stören and Rice, 1975), which artificially induces a vertex-like effect. However, with physically motivated micromechanical models, this destabilizing vertex effect is a natural outcome of crystal plasticity, which allows the occurrence of bifurcation without the need for enforcing any additional softening effects.

\section{Acknowledgements}

Part of this research has been performed within a project jointly funded by ArcelorMittal and CNRS. The first author is grateful to ArcelorMittal Research and CNRS for the financial support of his $\mathrm{PhD}$. 


\section{References}

Abed-Meraim, F., 1999. Sufficient conditions for stability of viscous solids. C. R. Acad. Sci. Série II b 327, 25-31.

Abed-Meraim, F., Nguyen, Q.S., 2007. A quasi-static stability analysis for Biot's equation and standard dissipative systems. European Journal of Mechanics - A/Solids 26, 383-393.

Anand, L., Kothari, M., 1996. A computational procedure for rate-independent crystal plasticity. J. Mech. Phys. Solids 44, 525-558.

Arminjon, M., 1991. A regular form of the Schmid law. Application to the ambiguity problem. Textures Microstruct. 14/18, 1121-1128.

Arul Kumar, M., Mahesh, S., 2012. Banding in single crystals during plastic deformation. Int. J. Plasticity 36, 15-33.

Asaro, R.J., 1979. Geometrical effects in the inhomogeneous deformation of ductile single crystals. Acta Metall. 27, 445-453.

Asaro, R.J., 1983. Crystal plasticity. J. Appl. Mech. 50, 921-934.

Asaro, R.J., Rice, J.R., 1977. Strain localization in ductile single crystals. J. Mech. Phys. Solids 25, 309-338.

Barbier, G., Benallal, A., Cano, V., 1998. Relation théorique entre la méthode de perturbation linéaire et l'analyse de bifurcation pour la prédiction de la localisation des déformations. C. R. Acad. Sci. Série II b 326, 153-158.

Barlat, F., 1987. Crystallographic Texture, Anisotropic Yield Surfaces and Forming Limits of Sheet Metals. Mat. Sci. Eng. 91, 55-72.

Bassani, J.L., Hutchinson, J.W., Neal, K.W., 1978. On the prediction of necking in anisotropic sheets. In: Lippmann, H., (Ed.), Proc. of IUTAM Symposium on Metal Forming Plasticity, Tutzing, Germany, Springer, Berlin, pp. 1-13.

Becker, R., 2011. An alternative approach to integrating plasticity relations. Int. J. Plasticity 27, 1224-1238.

Benallal, A., 2008. A note on ill-posedness for rate-dependent problems and its relation to the rateindependent case. Comput. Mech. 42, 261-269.

Berveiller, M., Fassi-Fehri, O., Hihi, A., 1987. The problem of two plastic and heterogeneous inclusions in an anisotropic medium. Int. J. Eng. Sci. 25 (6), 691-709. 
Berveiller, M., Zaoui, A., 1979. An extension of the self-consistent scheme to plastically-flowing polycrystals. J. Mech. Phys. Solids 26, 325-344.

Berveiller, M., Zaoui, A., 1984. Modeling of the plastic behaviour of inhomogeneous media. J. Eng. Mater. Technol. 106, 295-299.

Bigoni, D., Hueckel, T., 1990. On uniqueness and strain localization in plane strain and plane stress elastoplasticity. Mech. Res. Communications 17, 15-23.

Bigoni, D., Hueckel., T., 1991. Uniqueness and localization - I. Associative and non-associative elastoplasticity. Int. J. Solids Struct. 28, 197-213.

Bigoni, D., Zaccaria, D., 1992a. Strong ellipticity of comparison solids in elastoplasticity with volumetric non-associativity. Int. J. Solids Struct. 29, 2123-2136.

Bigoni, D., Zaccaria, D., 1992b. Loss of strong ellipticity in non-associative elastoplasticity. J. Mech. Phys. Solids 40, 1313-1331.

Bishop, J.F.W., Hill, R., 1951a. A theory of the plastic distortion of a polycristalline aggregate under combined stresses. Philos. Mag. 42, 414-427.

Bishop, J.F.W., Hill., R., 1951b. A theoretical derivation of the plastic properties of a polycristalline face-centred metal. Philos. Mag. 42, 1298-1307.

Boas, W., Schmid, E., 1934. Zur berechnung physikalischer konstanten quasi-isotroper vielkristalle. Helv. Phys. Acta. 7, 628-632.

Borg, U., 2007. Strain gradient crystal plasticity effects on flow localization. Int. J. Plasticity 23, 1400-1416.

Boudeau, N., Gelin, J.C., Salhi, S., 1998. Computational prediction of the localized necking in sheet forming based on microstructural material aspects. Comput. Mater. Sci. 11, 45-64.

Budiansky, B., 1965. On the elastic moduli of some heterogeneous materials. J. Mech. Phys. Solids $13,223-227$.

Budiansky, B., Wu, T.T., 1962. Theoretical prediction of plastic strain of polycrystals. Proc. $4^{\text {th }}$ U.S. Nat. Congr. Appl. Mech., 1175-1183.

Bunge, H.J., 1982. Texture Analysis in Materials Science. Butterworths, London.

Busso, E.P., Cailletaud, G., 2005. On the selection of active slip systems in crystal plasticity. Int. J.

Plasticity 21, 2212-2231.

Cayssials, F., 1998. A new method for predicting FLC. In: $20^{\text {th }}$ IDDRG Conference, Brussels. 
Cayssials, F., Lemoine, X., 2005. Predictive model of FLC (Arcelor model) upgraded to UHSS Steels. In: $24^{\text {th }}$ IDDRG Conference, Besançon.

Christoffersen, J., Hutchinson, J.W., 1979. A class of phenomenological corner theories of plasticity. J. Mech. Phys. Solids 27, 465-487.

Considère, A., 1885. Mémoire sur l'emploi du fer et de l'acier dans les constructions. Ann. Ponts et Chaussées 9, 574-775.

Dederichs, P.H., Zeller, R., 1973. Variational treatment of the elastic constants of disordered materials. Z. Phys. A Hadrons Nuclei 259, 103-116.

Drucker, D.C., 1950. Some implications of work hardening and ideal plasticity. Q. Appl. Math. 7, 411-418.

Drucker, D.C., 1956. On the uniqueness of the theory of stability. Q. Appl. Math. 16, 35-42.

Dudzinski, D., Molinari, A., 1991. Perturbation analysis of thermoviscoplastic instabilities in biaxial loading. Int. J. Solids Struct. 27 (5), 601-628.

Eshelby, J., 1957. The determination of the elastic field of an ellipsoidal inclusion and related problems. Proc. R. Soc. Lond. A 241, 376-396.

Essmann, U., Mughrabi., H., 1979. Annihilation of dislocations during tensile tests and cyclic deformation and limit of dislocations densities. Philos. Mag. A 40 (6), 731-756.

Fassi-Fehri, O., 1985. Le problème de la paire d'inclusions plastiques et hétérogènes dans une matrice anisotrope - Application à l'étude du comportement des matériaux composites et de la plasticité. PhD Thesis, Metz University, France.

Franciosi, P., 1984. Etude théorique et expérimentale du comportement élastoplastique de monocristaux métalliques se déformant par glissement : Modélisation pour un chargement complexe quasi-statique. PhD Thesis, Paris North University, France.

Franciosi, P., Zaoui, A., 1991. Crystal hardening and the issue of uniqueness. Int. J. Plasticity 7 , $295-311$.

Franz., G., 2008. Prédiction de la limite de formabilité des aciers multiphasés par une approche micromécanique. PhD Thesis, ENSAM Metz, France.

Franz, G., Abed-Meraim, F., Lorrain, J.P., Ben Zineb, T., Lemoine, X., Berveiller, M., 2009a. Ellipticity loss analysis for tangent moduli deduced from a large strain elastic-plastic self-consistent model. Int. J. Plasticity 25, 205-238. 
Franz, G., Abed-Meraim, F., Ben Zineb, T., Lemoine, X., Berveiller, M., 2009b. Strain localization analysis using a multiscale model. Comput. Mater. Sci. 45, 768-773.

Gambin, W., 1991. Crystal plasticity based on yield surfaces with rounded-off corners. Zeitschrift für Angewandte Mathematik und Mechanics, 71 (4), T265.

Gambin, W., 1992. Refined analysis of elastic-plastic crystals. Int. J. Solids Struct. 29 (16), 20132021.

Hadamard, J., 1903. Leçons sur la propagation des ondes et les équations de l'hydrodynamique. Chap. 6, Editions Hermann, Paris.

Haddad, A., 1997. Contribution à la détermination des courbes limites de formage en contrainte et en déformation à partir de la théorie $3 \mathrm{G}$. PhD Thesis, Savoie University, France.

Haddag, B., Abed-Meraim, F., Balan, T., 2009. Strain localization analysis using a large deformation elastic-plastic model coupled with damage. Int. J. Plasticity 25, 1970-1996.

Haddag, B., Balan, T., Abed-Meraim, F., 2007. Investigation of advanced strain-path dependent material models for sheet metal forming simulations. Int. J. Plasticity 23 (6), 951-979.

Harewood, F.J., McHugh, P.E., 2006. Investigation of finite element mesh independence in rate dependent materials. Comp. Mater. Sci. 37, 442-453.

Harren, S.V., Asaro, R.J., 1989. Nonuniform deformations in polycrystals and aspects of the validity of the Taylor model. J. Mech. Phys. Solids 37, 191-232.

Hershey, A.V., 1954. The elasticity of an isotropic aggregate of anisotropic cubic crystals. J. Appl. Mech. 21, 236-240.

Hill, R., 1952. On discontinuous plastic states, with special reference to localized necking in thin sheets. J. Mech. Phys. Solids 1, 19-30.

Hill, R., 1958. A general theory of uniqueness and stability in elastic-plastic solids. J. Mech. Phys. Solids 6, 239-249.

Hill, R., 1962. Acceleration waves in solids. J. Mech. Phys. Solids 10, 1-16.

Hill, R., 1965a. Continuum micro-mechanics of elastoplastic polycrystals. J. Mech. Phys. Solids 13, 89-101.

Hill, R., 1965b. A self consistent mechanics of composite materials. J. Mech. Phys. Solids 13, 213221.

Hill, R., 1966. Generalized constitutive relations for incremental deformation of metal crystals by multislip. J. Mech. Phys. Solids 14, 95-102. 
Hill, R., 1967. The essential structure of constitutive laws for metal composites and polycrystals. J. Mech. Phys. Solids 15, 79-95.

Hill, R., 1972. On constitutive macrovariables for heterogeneous solids at finite strain. Proc. R. Soc. Lond. A 326, 131-141.

Hill, R., Hutchinson, J.W., 1975. Bifurcation phenomena in the plane tension test. J. Mech. Phys. Solids 23, 239-264.

Hill, R., Rice, J.R., 1972. Constitutive analysis of elastic-plastic crystals at arbitrary strain. J. Mech. Phys. Solids 20, 401-413.

Hiwatashi, S., Van Bael, A., Van Houtte, P., Teodosiu, C., 1998. Predictions of forming limit strains under strain-path changes: applications of an anisotropic model based on texture and dislocation structure. Int. J. Plasticity 14, 647-669.

Hoc, T., 1999. Etudes expérimentales et numériques de la localisation de la déformation dans les aciers doux. PhD Thesis, Ecole Centrale de Paris, France.

Hora, P., Tong, L., Reissner, J., 1996. A prediction method of ductile sheet metal failure in FE simulation. In: Wagonner, R., et al. (Eds.), Proc. of Numisheet'96, pp. 252-256.

Horstemeyer, M.F., 2000. A numerical parametric investigation of localization and forming limits. Int. J. Damage Mech. 9, 255-285.

Hughes, T.J.R., 1984. Numerical implementation of constitutive models: rate-independent deviatoric plasticity. In: Theoretical Foundation for Large-scale Computations for Nonlinear Material Behavior. Martinus Nij Publishers, Netherlands, pp. 29-57.

Hutchinson, J.W., 1970. Elastic plastic behaviour of polycrystalline metals and composites. Proc. R. Soc. Lond. A 319, 247-272.

Hutchinson, J.W., 1974. Plastic buckling. In: Yih, C.-S., (Ed.), Adv. Appl. Mech., vol.14. Academic Press, New York, pp. 67-144.

Hutchinson, J.W., Neale, K.W., 1978. Sheet necking - II. Time-independent behavior. In:

Koistinen, D.P., Wang, N.M. (Eds.), Mechanics of Sheet Metal Forming. Plenum Press, New York, pp. 127-153.

Hutchinson, J.W., Tvergaard, V., 1981. Shear band formation in plane-strain. Int. J. Solids Struct. $17,451-470$.

Inal, K., Neale, K.W., Aboutajeddine, A., 2005. Forming limit comparisons for FCC and BCC sheets. Int. J. Plasticity 21, 1255-1266. 
Iwakuma, T., Nemat-Nasser, S., 1984. Finite elastic-plastic deformation of polycrystalline metals and composite. Proc. R. Soc. Lond. A 394, 87-119.

Keh, A.S., Nakada, Y., 1967. Plasticity of iron single crystal. Can. J. Phys. 45, 1101-1120.

Kim, J.H., Lee, M.-G., Barlat, F., Wagoner, R.H., Chung, K., 2008. An elasto-plastic constitutive model with plastic strain rate potentials for anisotropic cubic metals. Int. J. Plasticity 24, 22982334.

Knockaert, R., Chastel, Y., Massoni, E., 2002. Forming limits prediction using rate-independent polycrystalline plasticity. Int. J. Plasticity 18, 231-247.

Kobayashi, M., 2010. Analysis of deformation localization based on proposed theory of ultrasonic wave velocity propagating in plastically deformed solids. Int. J. Plasticity 26, 107-125.

Kocks, U.F., 1976. Laws for work-hardening and low-temperature creep. J. Eng. Mat. Tech. 98, 7685.

Kröner, E., 1958. Berechnung der elastischen konstanten des vielkristalls aus den kontanten des einkristalls. Z. Phys. 151, 504-518.

Kröner, E., 1961. Zur plastischen verformung des vielkristalls. Acta Metall. 9, 155-165.

Kuroda, M., Tvergaard, V., 1999. Use of abrupt strain path change for determining subsequent yield surface: illustrations of basic idea. Acta Materialia 47, 3879-3890.

Kuroda, M., Tvergaard, V., 2001a. A phenomenological plasticity model with non-normality effects representing observations in crystal plasticity. J. Mech. Phys. Solids 49, 1239-1263.

Kuroda, M., Tvergaard, V., 2001b. Shear band development predicted by a non-normality theory of plasticity and comparison to crystal plasticity predictions. Int. J. Solids Struct. 38, 8945-8960.

Kuwabara, T., 2007. Advances in experiments on metal sheets and tubes in support of constitutive modeling and forming simulations. Int. J. Plasticity 23, 385-419.

Kuwabara, T., Kuroda, M., Tvergaard, V., Nomura K., 2000. Use of abrupt strain path change for determining subsequent yield surface: experimental study with metal sheets. Acta Materialia 48, 2071-2079.

Lele, S.P., Anand, L., 2009. A large-deformation strain-gradient theory for isotropic viscoplastic materials. Int. J. Plasticity 25, 420-453.

Li, Y., Karr, D.G., 2009. Prediction of ductile fracture in tension by bifurcation, localization, and imperfection analyses. Int. J. Plasticity 25, 1128-1153. 
Lian, J., Barlat, F., Baudelet, B., 1989. Plastic behaviour and stretchability of sheet metals. II. Effect of yield surface shape on sheet forming limit. Int. J. Plasticity 5, 131-147.

Lin, T.H., 1957. Analysis of elastic and plastic strains of a face-centered cubic crystal. J. Mech. Phys. Solids 5, 143-149.

Lin, T.H., 1971. Physical theory of plasticity. In: Yih, C.S. (Ed.), Advances in Applied Mechanics 11. Academic Press, New-York, pp. 255-311.

Lipinski, P., Berveiller, M., 1989. Elastoplasticity of micro-inhomogeneous metals at large strains. Int. J. Plasticity 5, 149-172.

Lipinski, P., Berveiller, M., Reubrez, E., Morreale, J., 1995. Transition theories of elastic-plastic deformation of metallic polycrystals. Arch. Appl. Mech. 65, 291-311.

Lorrain, J.P., 2005. Critère de ductilité basé sur la perte d'ellipticité du module tangent élastoplastique déduit d'un modèle autocohérent. PhD Thesis, ENSAM Metz, France.

Loret, B., Rizzi, E., 1997a. Anisotropic stiffness degradation triggers the onset of strain localization. Int. J. Plasticity 13, 447-459.

Loret, B., Rizzi, E., 1997b. Qualitative analysis of strain localization. Part II: Transversely isotropic elasticity and plasticity. Int. J. Plasticity 13, 501-519.

Luft., A. 1991. Microstructural processes of plastic instabilities in strengthened metals. Prog. Mater. Sci. 35, 97-204.

Lyapunov, A., 1892. The general problem of stability of motion. Engl. Transl. (1992), Taylor and Francis, London.

Malvern, L.E., 1969. Introduction to the mechanics of a continuous medium. Englewood Cliffs, Prentice-Hall, New Jersey.

Mandel, J., 1966. Conditions de stabilité et postulat de Drucker. In: J. Kravtchenko, J., Sirieys, P.M. (Eds.), Rheology and soil mechanics. Springer-Verlag, pp. 58-68.

Marciniak, Z., Kuczynski, K., 1967. Limit Strains in processes of stretch-forming sheet metal. Int. J. Mech. Sci. 9 (9), 609-620.

Mattiasson, K., Sigvant, M., Larson, M., 2006. Methods for forming limit prediction in ductile metal sheets. In: Santos, A.D., Barata da Rocha, A. (Eds.), Proc. IDDRG’06. Porto, pp. 1-9.

Molinari, A., Clifton, R., 1987. Analytical characterization of shear localization in thermo-viscoplastic solids. J. Appl. Mech. 54, 806-812. 
Mroginski, J.L., Etse, G., Vrech, S.M., 2011. A thermodynamical gradient theory for deformation and strain localization of porous media. Int. J. Plasticity 27, 620-634.

Neale, K.W., Chater, E., 1980. Limit strain predictions for strain-rate sensitive anisotropic sheets. Int. J. Mech. Sci. 22, 563-574.

Needleman, A., Tvergaard, V., 1977. Necking of biaxially stretched elastic-plastic circular plates. J. Mech. Phys. Solids 25, 159-183.

Needleman, A., Tvergaard, V., 1992. Analyses of plastic flow localization in metals. App. Mech. Rev. 45, S3-S18.

Neilsen, M.K., Schreyer, H.L., 1993. Bifurcations in elastic-plastic materials. Int. J. Solids Struct. $30,521-544$.

Nemat-Nasser, S., Mehrabadi, M.M., Iwakuma, T., 1981. On certain macroscopic and microscopic aspects of plastic flow of ductile materials. In: Nemat-Nasser, S. (Ed.), Three-dimensional constitutive relations and ductile fracture. North-Holland, Amsterdam, pp. 157-172.

Nesterova, E.V., Bacroix, B., Teodosiu, C., 2001. Microstructure and texture evolution under strain path changes in low-carbon interstitial-free steel. Met. Mat. Trans. A 32A, 2527-2538.

Ortiz, M., Popov, E.P., 1985. Accuracy and stability of integration algorithms for elastoplastic constitutive relations. Int. J. Numer. Methods Engng. 21, 1561-1576.

Peeters, B., 2002. Multiscale modelling of the induced plastic anisotropy in IF steel during sheet forming. PhD Thesis, Katholieke Universiteit Leuven, Belgium.

Peeters, B., Seefeldt, M., Van Houtte, P., Aernoudt, E., 2001. Taylor ambiguity in BCC polycrystals: a non-problem if substructural anisotropy is considered. Scripta Mater. 45, 13491356.

Peirce, D., 1983. Shear band bifurcations in ductile single crystals J. Mech. Phys. Solids 31 (2), $133-153$.

Peirce, D., Asaro, R.J., Needleman, A., 1982. An analysis of nonuniform and localized deformation in ductile single crystals. Acta metall. 30, 1087-1119.

Peirce, D., Asaro, R.J., Needleman, A., 1983. Material rate dependence and localized deformation in crystalline solids. Acta metall. 31 (12), 1951-1976.

Pollnow, D., Pennelle, R., Lacombe, D., 1972. Étude des propriétés mécaniques et du taux de consolidation de monocristaux de fer déformés à température ambiante. Mémoires Scientifiques Revue Métallurgique 10, 703-714. 
Rauch, E.F., 1998. Plastic anisotropy of sheet metals determined by simple shear tests. Mat. Sci. Eng. A 241, 179-183.

Reuss, A., 1929. Berechnung der fliessgrenze von mischkristallen auf grund der plastizitätsbedingung für einkristalle. Z. Angew. Math. Mech. 9, 49-58.

Rice, J.R., 1976. The localization of plastic deformation. In: $14^{\text {th }}$ International Congress of Theoretical and Applied Mechanics, pp. 207-220.

Rizzi, E., Loret, B., 1997. Qualitative analysis of strain localization. Part I: Transversely isotropic elasticity and isotropic plasticity. Int. J. Plasticity 13, 461-499.

Rudnicki, J.W., Rice, J.R., 1975. Conditions for the localization of deformation in pressuresensitive dilatant materials. J. Mech. Phys. Solids 23, 371-394.

Sachs, G., 1928. Zur abteilung einer fleissbedingung. Zeit. Der V.D.I. 72, 739-747.

Saje, M., Pan, J., Needleman, A., 1982. Void nucleation effects on shear localization in porous plastic solids. Int. J. Fracture 19, 163-182.

Sánchez, P.J., Huespe, A.E., Oliver, J., 2008. On some topics for the numerical simulation of ductile fracture. Int. J. Plasticity 24, 1008-1038.

Sewell, M.J., 1973. Yield-surface corner lowers buckling stress of an elastic-plastic plate under compression. J. Mech. Phys. Solids 21, 19-45.

Sewell, M.J., 1974. Plastic-flow rule at a yield vertex. J. Mech. Phys. Solids 22, 469-490.

Shanthraj, P., Zikry, M.A., 2012. Dislocation-density mechanisms for void interactions in crystalline materials. Int. J. Plasticity 34, 154-163.

Signorelli, J.W., Bertinetti, M.A., Turner, P.A., 2009. Predictions of forming limit diagrams using a rate-dependent polycrystal self-consistent plasticity model. Int. J. Plasticity 25, 1-25.

Signorelli, J.W., Serenelli, M.J., Bertinetti, M.A., 2012. Experimental and numerical study of the role of crystallographic texture on the formability of an electro-galvanized steel sheet. J. Mat. Proc. Tech. 212, 1367-1376.

Simo, J., Taylor, R., 1985. Consistent tangent operators for rate-independent elasto-plasticity. Comput. Methods Appl. Mech. Eng. 48, 101-118.

Stören, S., Rice, J.R., 1975. Localized necking in thin sheets. J. Mech. Phys. Solids 23, 421-441. Stoughton, T.B., Zhu, X., 2004. Review of theoretical models of the strain-based FLD and their relevance to the stress-based FLD. Int. J. Plasticity 20, 1463-1486. 
Sun, X., Choi, K.S., Liu, W.N., Khaleel, M.A., 2009. Predicting failure modes and ductility of dual phase steels using plastic strain localization. Int. J. Plasticity 25, 1888-1909.

Swift, H.W., 1952. Plastic instability under plane stress. J. Mech. Phys. Solids 1, 1-18.

Tabourot, L., 1992. Loi de comportement élastoviscoplastique du monocristal en grandes déformations. PhD Thesis, Grenoble University, France.

Takahashi, H., 1988. Predictions of plastic stress-strain relations of polycrystals based on the Lin model. Int. J. Plasticity 4, 231-250.

Tadano, Y., Kuroda, M., Noguchi, H., 2012. Quantitative re-examination of Taylor model for FCC polycrystals. Comput. Mater. Sci. 21, 290-302.

Taylor, G.I., 1934. The mechanism of plastic deformation of crystals. Proc. R. Soc. Lond. A 145, $362-387$.

Taylor, G.I., 1938. Plastic strain in metals. J. Inst. Metals 62, 307-315.

Teodosiu, C., Hu, Z., 1998. Microstructure in the continuum modeling of plastic anisotropy. In:

Proc. $19^{\text {th }}$ Riso International Symposium on Materials Science. Roskilde, Denmark, pp. 149-168.

Thomas, T.Y., 1961. Plastic flow and fracture in solids. Academic Press.

Toth, L.S., Duzinski, D., Molinari, A., 1995. Forming limit predictions with the perturbation method using stress potential functions of polycrystal viscoplasticity. Int. J. Mech. Sci. 38, 805824.

Truesdell, C., Noll, W., 1965. The non-linear field theories of mechanics. Encyclopedia of Physics, vol. III/3, Springer-Verlag, Berlin.

Valanis, K.C., 1989. Banding and stability in plastic materials. Acta Mech. 79, 113-141.

Viatkina, E.M., Brekelmans, W.A.M., Geers, M.G.D., 2005. A crystal plasticity based estimate for forming limit diagrams from textural inhomogeneities. J. Mat. Proc. Tech. 168, 211-218.

Voigt, W., 1889. Über die beziehung zwischen den beiden elastizitäts-konstanten isotroper körper. Wied. Ann. 38, 573-587.

Weng, G.J., 1980. Constitutive equations of single crystals and polycristalline aggregates under cyclic loading. J. Eng. Mat. Technol. 18, 1385-1397.

Willis, J.R., 1988. (Private communication).

Wu, P.D., Graf, A., MacEwen, S.R., Lloyd, D.J., Jain, M., Neale, K.W., 2005. On forming limit stress diagram analysis. Int. J. Solids Struct. 42, 2225-2241. 
Wu, P.D., Lloyd, D.J., Jain, M., Neale K.W., Huang, Y., 2007. Effects of spatial grain orientation distribution and initial surface topography on sheet metal necking. Int. J. Plasticity 23, 1084-1104.

Wu, P.D., Neale, K.W., Van der Giessen, E., 1997. On crystal plasticity FLD analysis. Proc. R. Soc. Lond. A 453, 1831-1848.

Yamamoto, H., 1978. Conditions for shear localization in the ductile fracture of void-containing materials. Int. J. Fracture 14, 347-365.

Yoshida, K., Brenner, R., Bacroix, B., Bouvier, S., 2009. Effect of regularization of Schmid law on self-consistent estimates for rate-independent plasticity of polycrystals. European Journal of Mechanics - A/Solids 28, 905-915.

Yoshida, K., Ishizaka, T., Kuroda, M., Ikawa, S., 2007. The effects of texture on formability of aluminum alloy sheets. Acta Materialia 55, 4499-4506.

Yoshida, K., Kuroda, M., 2012a. Comparison of bifurcation and imperfection analyses of localized necking in rate-independent polycrystalline sheets. Int. J. Solids Struct. 49, 2073-2084.

Yoshida, K., Kuroda, M., 2012b. Numerical investigation on a key factor in superior stretchability of face-centered cubic polycrystalline sheets. Int. J. Mech. Sci. 58, 47-56.

Zhang, F., Bower, A.F., Mishra, R.K., Boyle, K.P., 2009. Numerical simulations of necking during tensile deformation of aluminum single crystals. Int. J. Plasticity 25, 49-69.

Zhao, Z., Ramesh, M., Raabe, D., Cuitino, A.M., Radovitzky, R., 2008. Investigation of threedimensional aspects of grain-scale plastic surface deformation of an aluminum oligocrystal. Int. J. Plasticity 24, 2278-2297.

Zhou, Y., Neale, K.W., 1995. Predictions of forming limit diagrams using a rate-sensitive crystal plasticity model. Int. J. Mech. Sci. 37, 1-20. 
Table 1. Material parameters and initial crystallographic orientation used for the investigation of the effect of certain physical parameters on the ductility limit of single crystals

\begin{tabular}{cccccccccc}
\hline Parameters & $\tau_{c 0}\{110\}$ & $\tau_{c 0}\{112\}$ & $\rho_{0}$ & $\varphi_{1_{0}}$ & $\phi_{0}$ & $\varphi_{2_{0}}$ & $g_{0}$ & $y_{c}$ & $D$ \\
\hline Values & $55 \mathrm{MPa}$ & $50 \mathrm{MPa}$ & $10^{9} \mathrm{~m}^{-2}$ & $148^{\circ}$ & $128^{\circ}$ & $156^{\circ}$ & 80 & $2 \mathrm{~nm}$ & $1 \mathrm{~mm}$ \\
\hline
\end{tabular}


Table 2. Material parameters used for the investigation of the effect of certain physical parameters on the ductility limit of polycrystals

\begin{tabular}{cccccc}
\hline Parameters & $\tau_{c 0}\{110\}$ & $\tau_{c 0}\{112\}$ & $g_{0}$ & $y_{c}$ & $D$ \\
\hline Values & $55 \mathrm{MPa}$ & $55 \mathrm{MPa}$ & 90 & $3.25 \mathrm{~nm}$ & $20 \mu \mathrm{m}$ \\
\hline
\end{tabular}


Fig. 1. Schematic representation of the multiplicative decomposition of the deformation gradient: plastic slip and rigid rotation along with elastic distortion of the crystallographic lattice

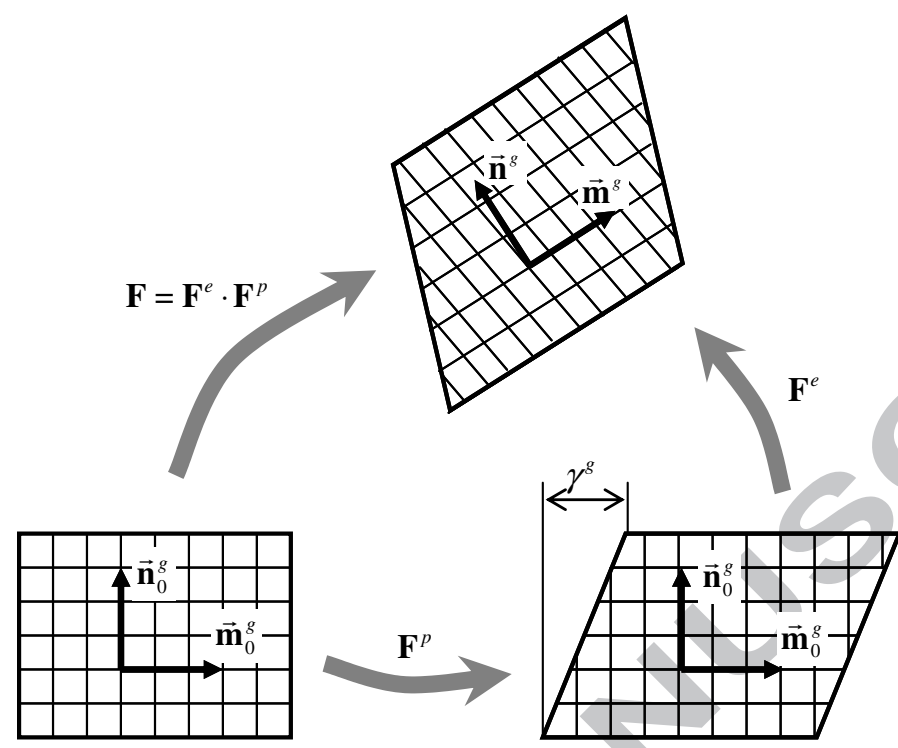


Fig. 2. Illustration of the velocity jump of certain mechanical fields across the band plane with respect to a fixed reference configuration (left) and relative to the current reference configuration (right)
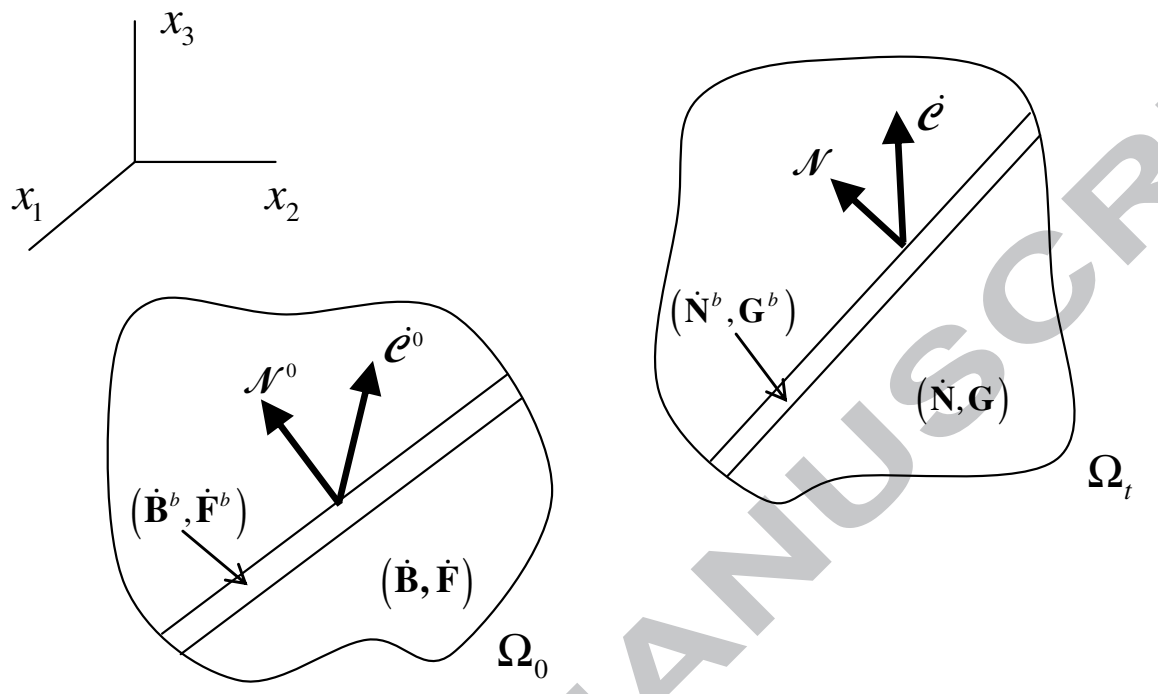
Fig. 3. Inverse pole figure of the studied single crystal: Orientation of the RD (a), TD

(b) and ND (c) relative to the crystal axes

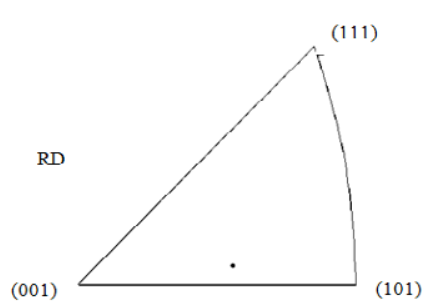

(a)

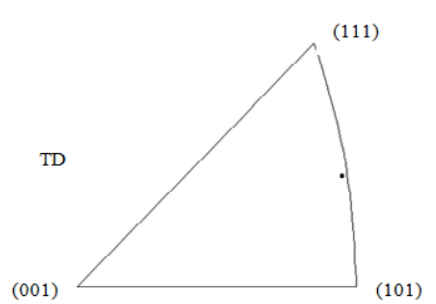

(b)

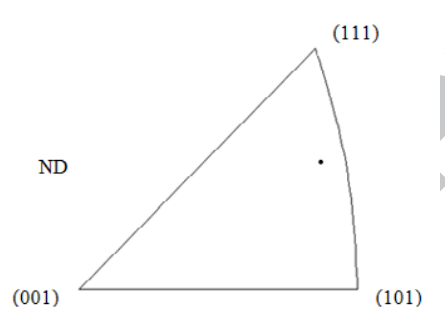

(c) 
Fig. 4. Effect of the initial critical shear stress (of $\{110\}$ and $\{112\}$ slip plane families) on the ductility limit of a single crystal: Responses for uniaxial tensile tests performed parallel to the rolling direction until the loss of ellipticity (top) and the minimal determinant of the acoustic tensor over all orientations of the normal to the localization band (bottom)
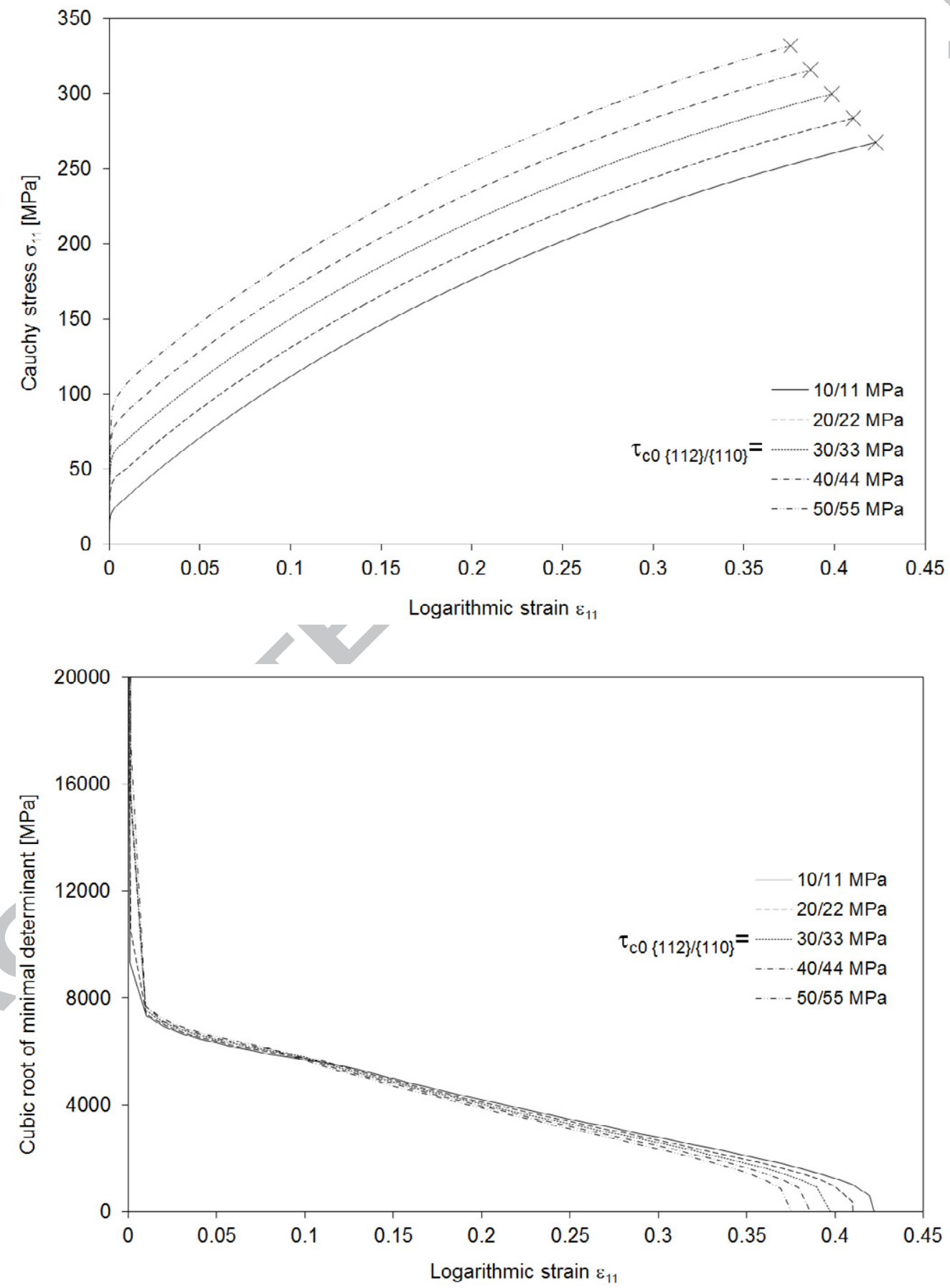
Fig. 5. Effect of the initial critical shear stress (of $\{110\}$ and $\{112\}$ slip plane families) on the ductility limit of a single crystal: Responses for uniaxial tensile tests performed parallel to the rolling direction until the loss of strong ellipticity (top) and the minimal eigenvalue of the symmetric part of the acoustic tensor over all orientations of the normal to the localization band (bottom)
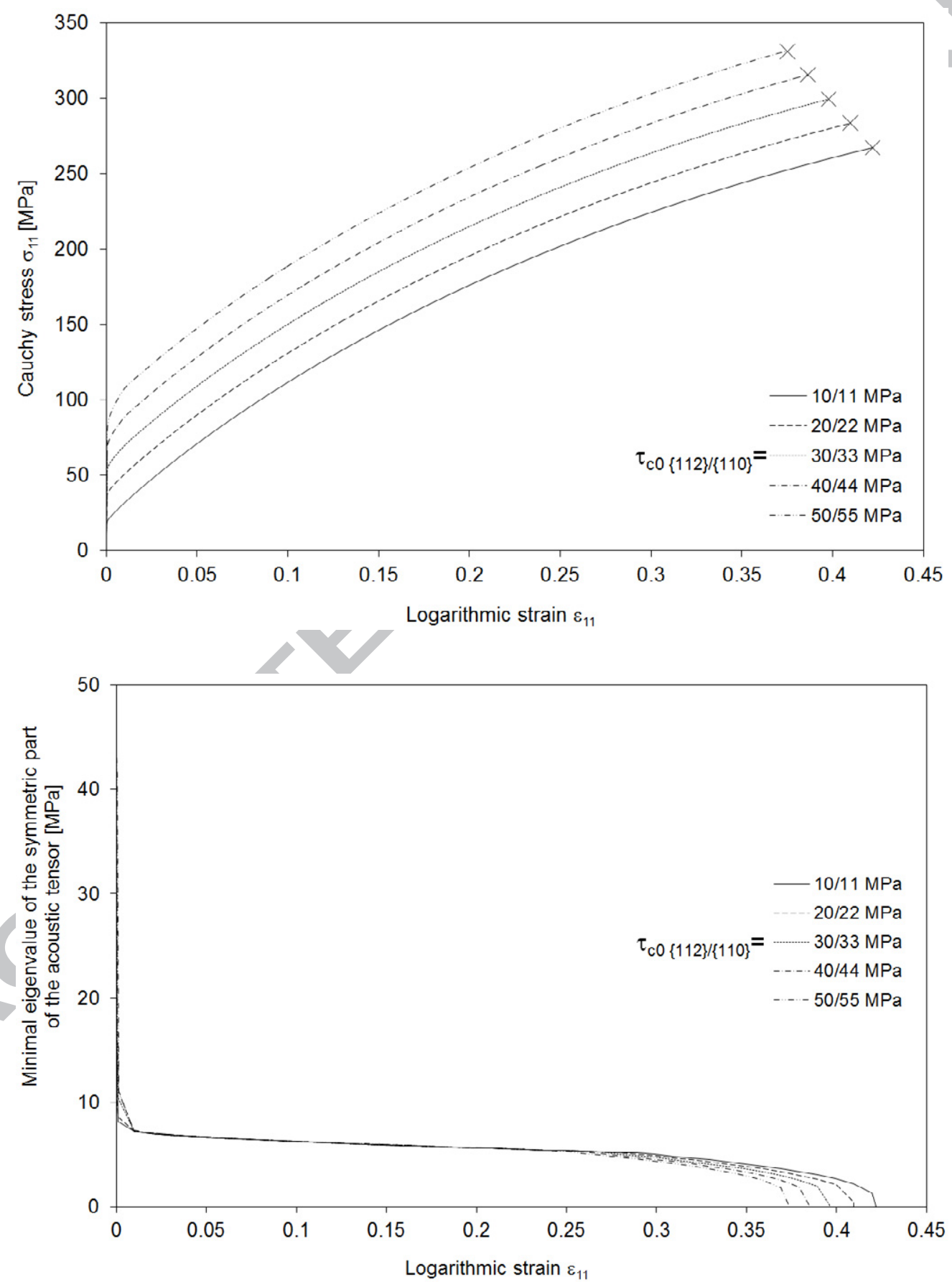
Fig. 6. Effect of the critical annihilation distance of dislocations on the ductility limit of a single crystal: Responses for uniaxial tensile tests performed parallel to the rolling direction until the loss of ellipticity (top) and the minimal determinant of the acoustic tensor over all orientations of the normal to the localization band (bottom)
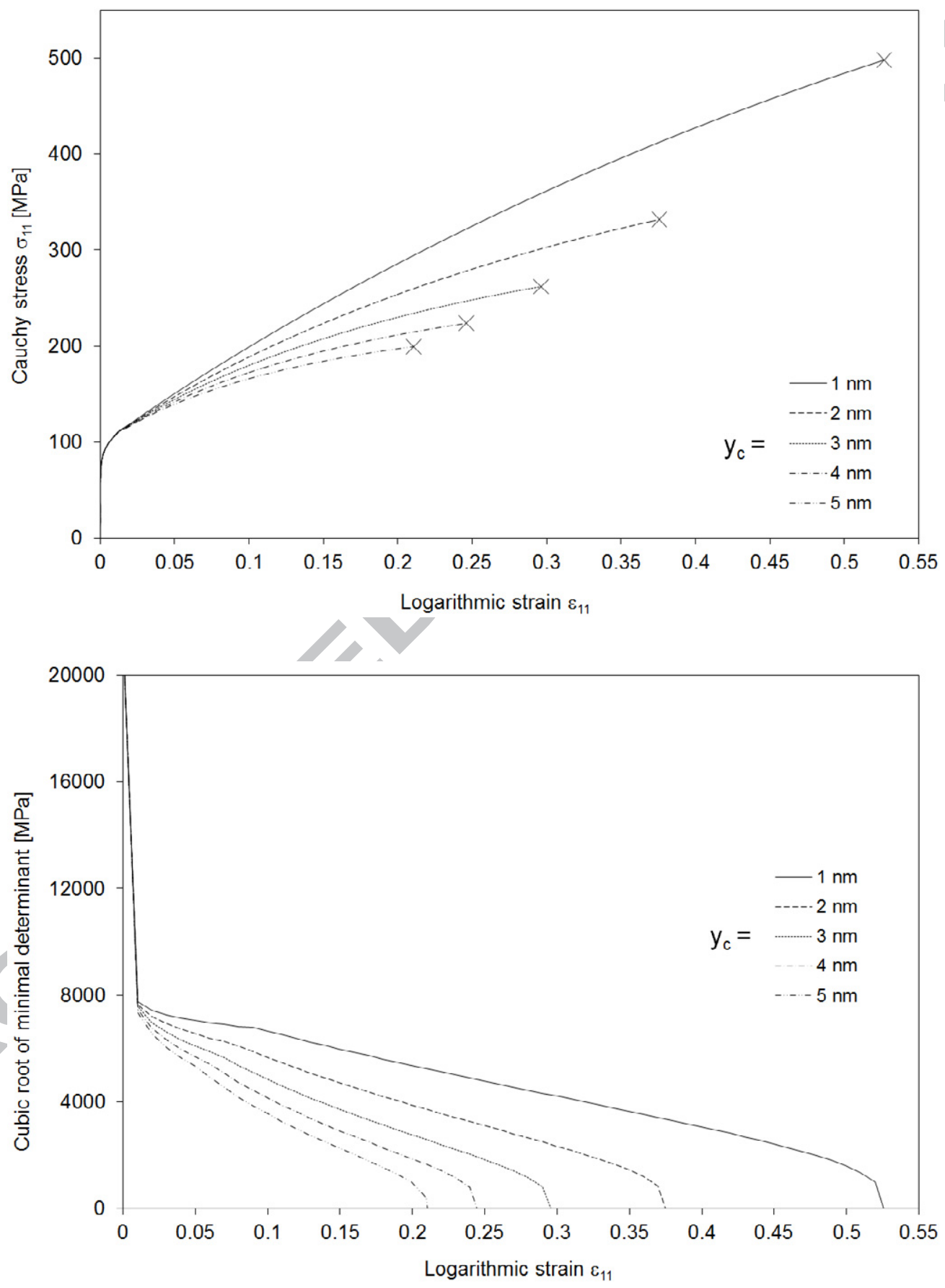
Fig. 7. Effect of the mean free path parameter on the ductility limit of a single crystal: Responses for uniaxial tensile tests performed parallel to the rolling direction until the loss of ellipticity (top) and the minimal determinant of the acoustic tensor over all orientations of the normal to the localization band (bottom)
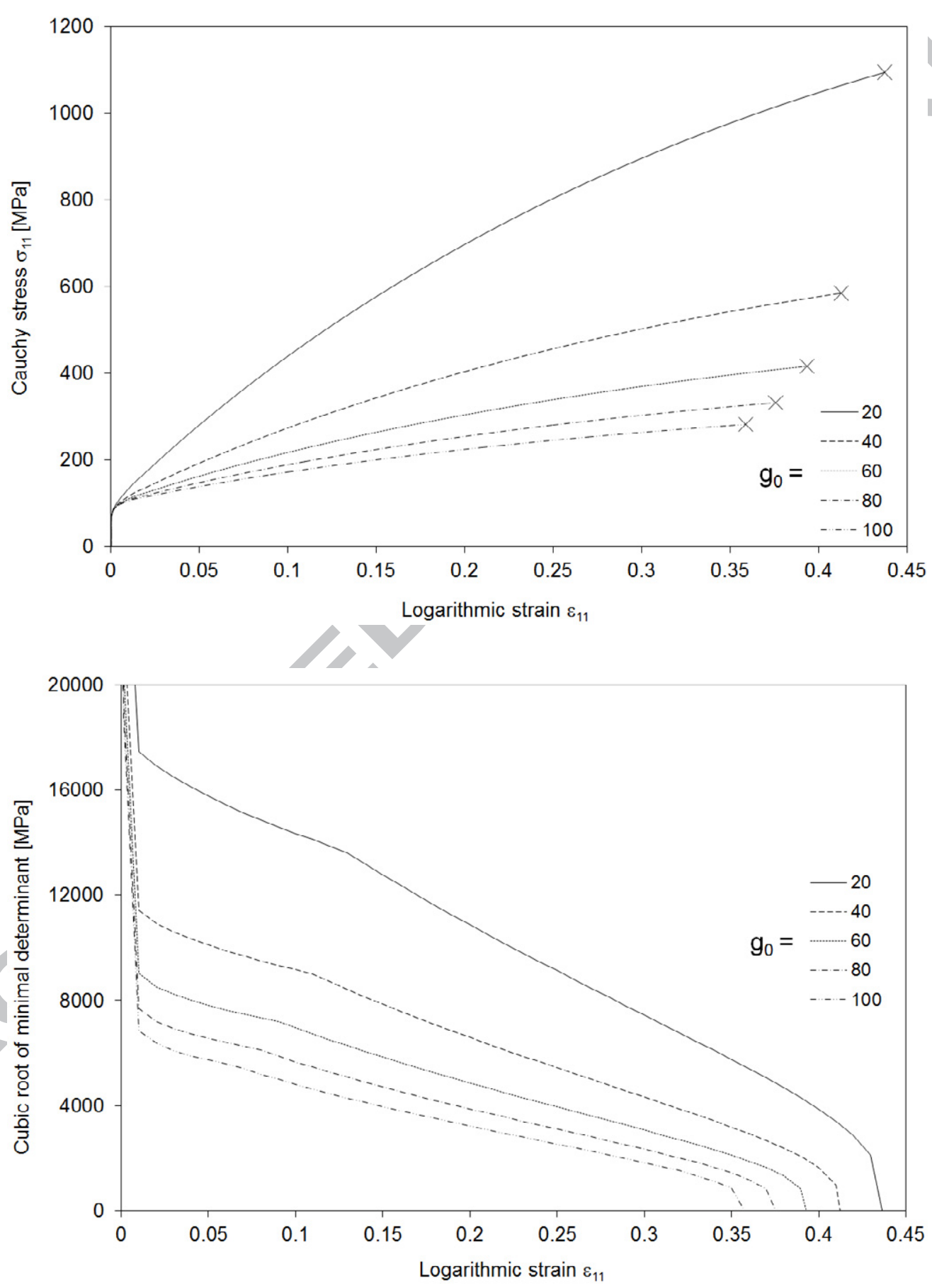
Fig. 8. Effect of the initial critical shear stress (of $\{110\}$ and $\{112\}$ slip plane families) on the ductility limit of a single crystal: Responses for uniaxial tensile tests performed parallel to the transverse direction until the loss of ellipticity (top) and the minimal determinant of the acoustic tensor over all orientations of the normal to the localization band (bottom)
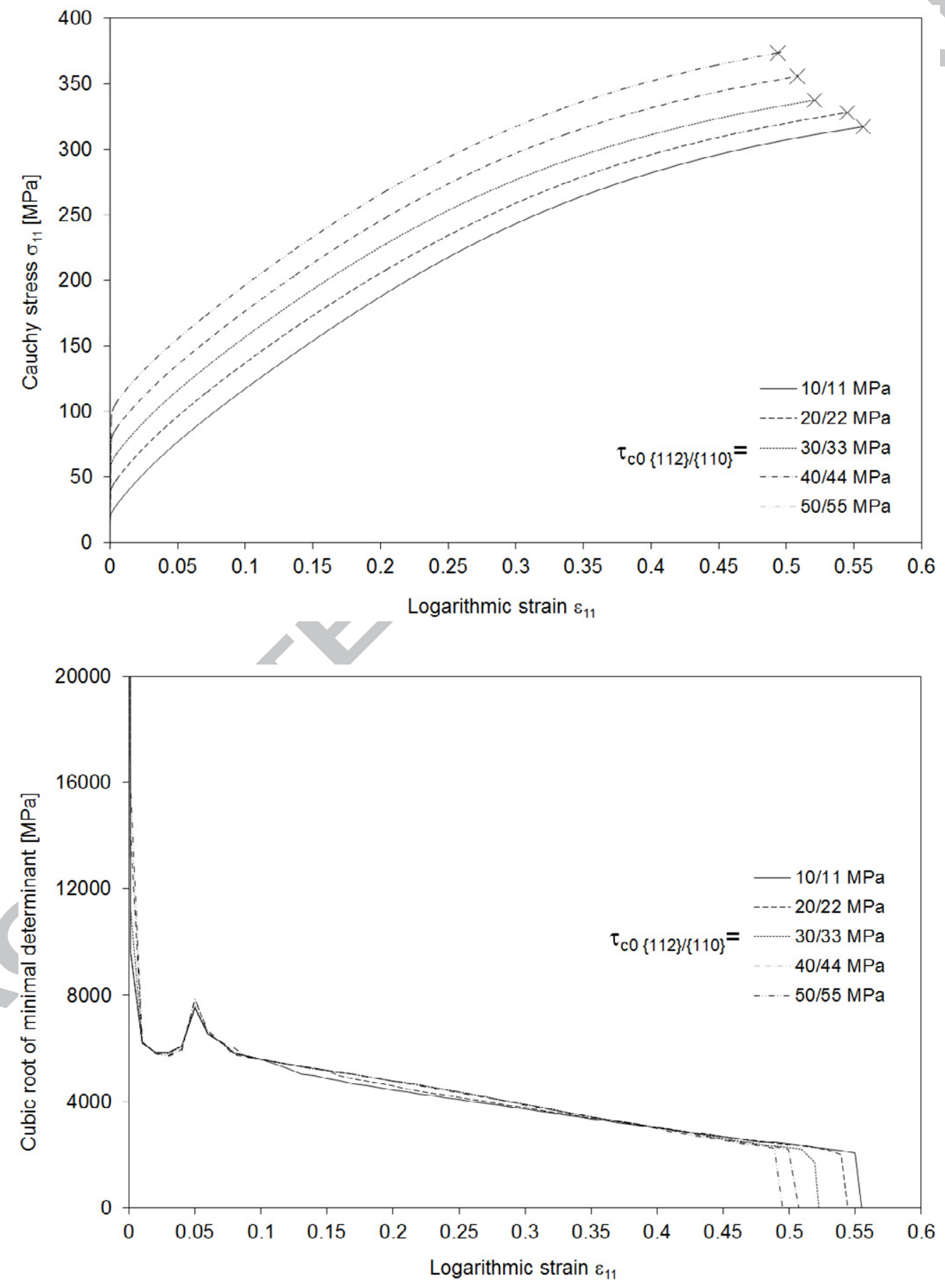
Fig. 9. Effect of the critical annihilation distance of dislocations on the ductility limit of a single crystal: Responses for uniaxial tensile tests performed parallel to the transverse direction until the loss of ellipticity (top) and the minimal determinant of the acoustic tensor over all orientations of the normal to the localization band (bottom)
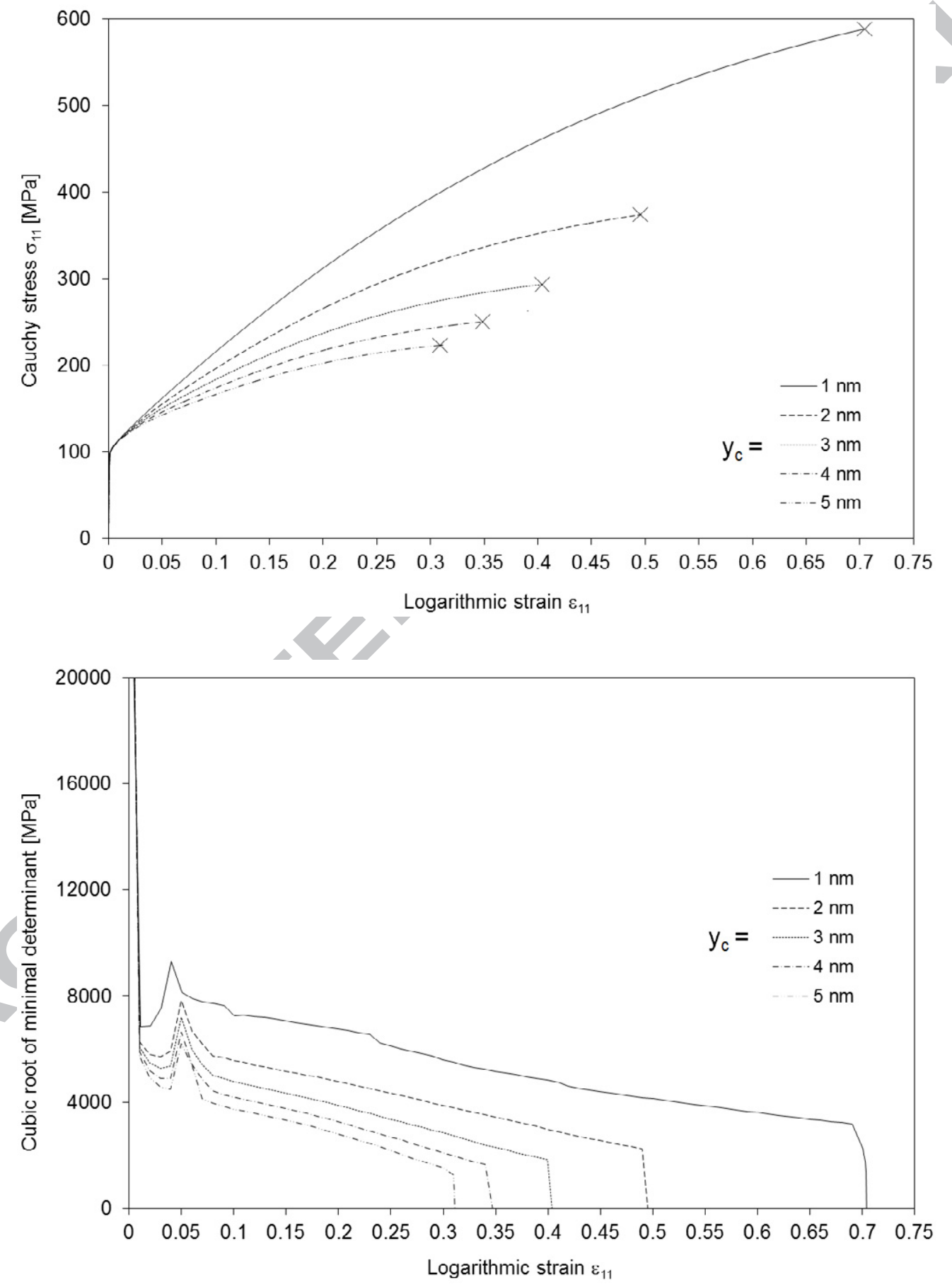
Fig. 10. Effect of the mean free path parameter on the ductility limit of a single crystal: Responses for uniaxial tensile tests performed parallel to the transverse direction until the loss of ellipticity (top) and the minimal determinant of the acoustic tensor over all orientations of the normal to the localization band (bottom)
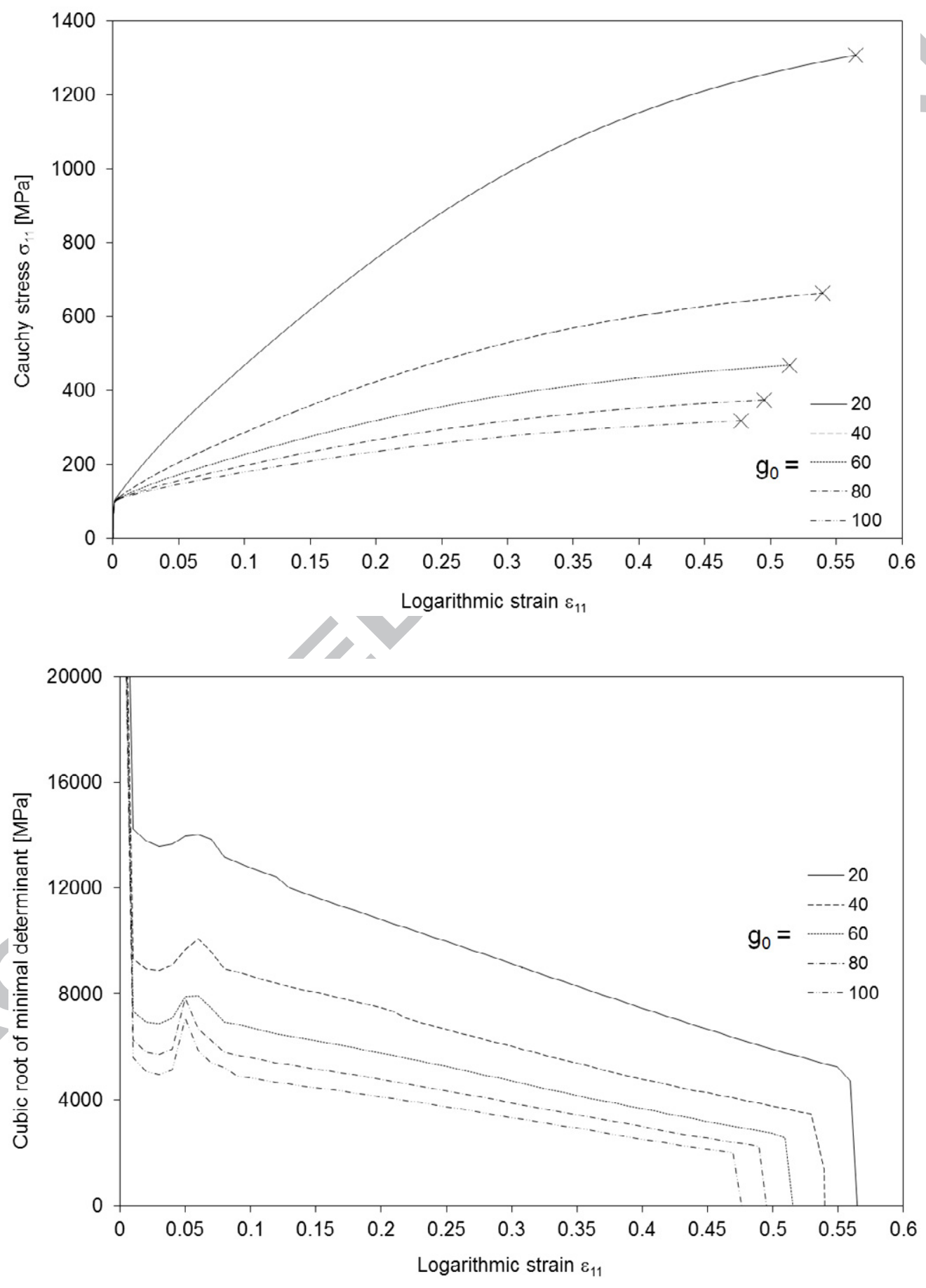
Fig. 11. Initial random texture (1000 grains) of the studied IF-Ti single-phase steel: (a) $\{100\}$-pole figure, (b) $\{110\}$-pole figure

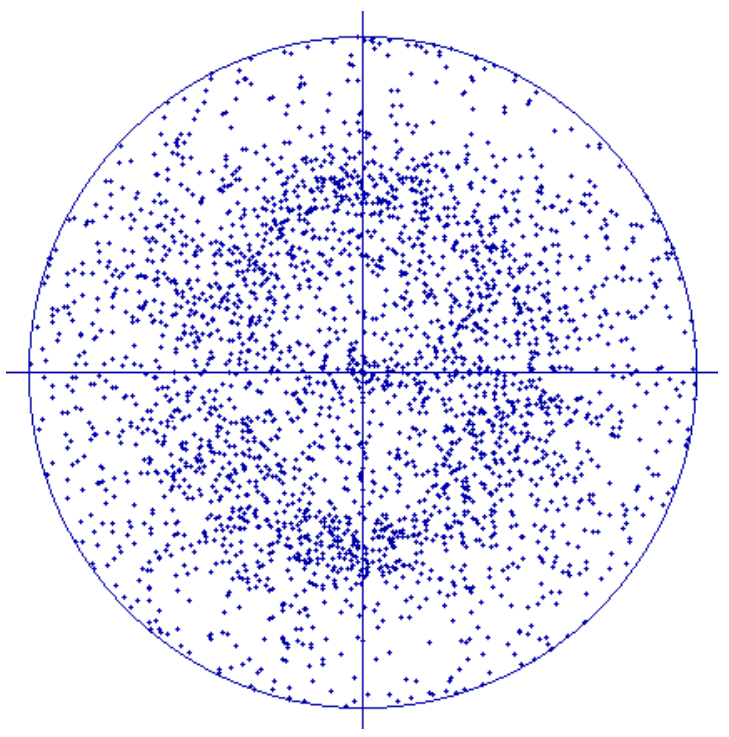

(a)

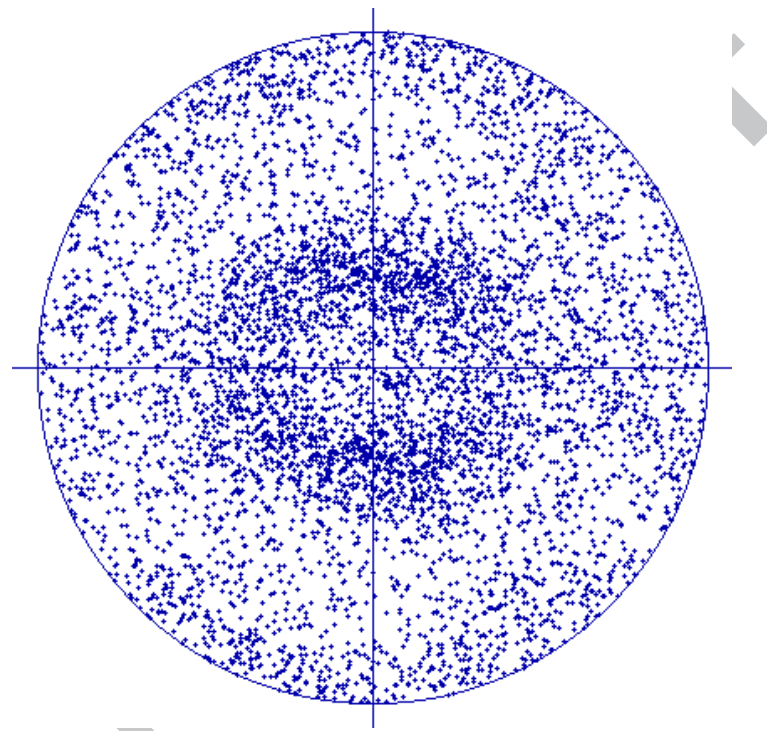

(b) 
Fig. 12. Effect of the initial critical shear stress (of $\{110\}$ and $\{112\}$ slip plane families) on the ductility limit of a polycrystal (self-consistent scheme): Responses for plane strain tensile tests performed parallel to the rolling direction until the loss of ellipticity (top) and the minimal determinant of the acoustic tensor over all orientations of the normal to the localization band (bottom)
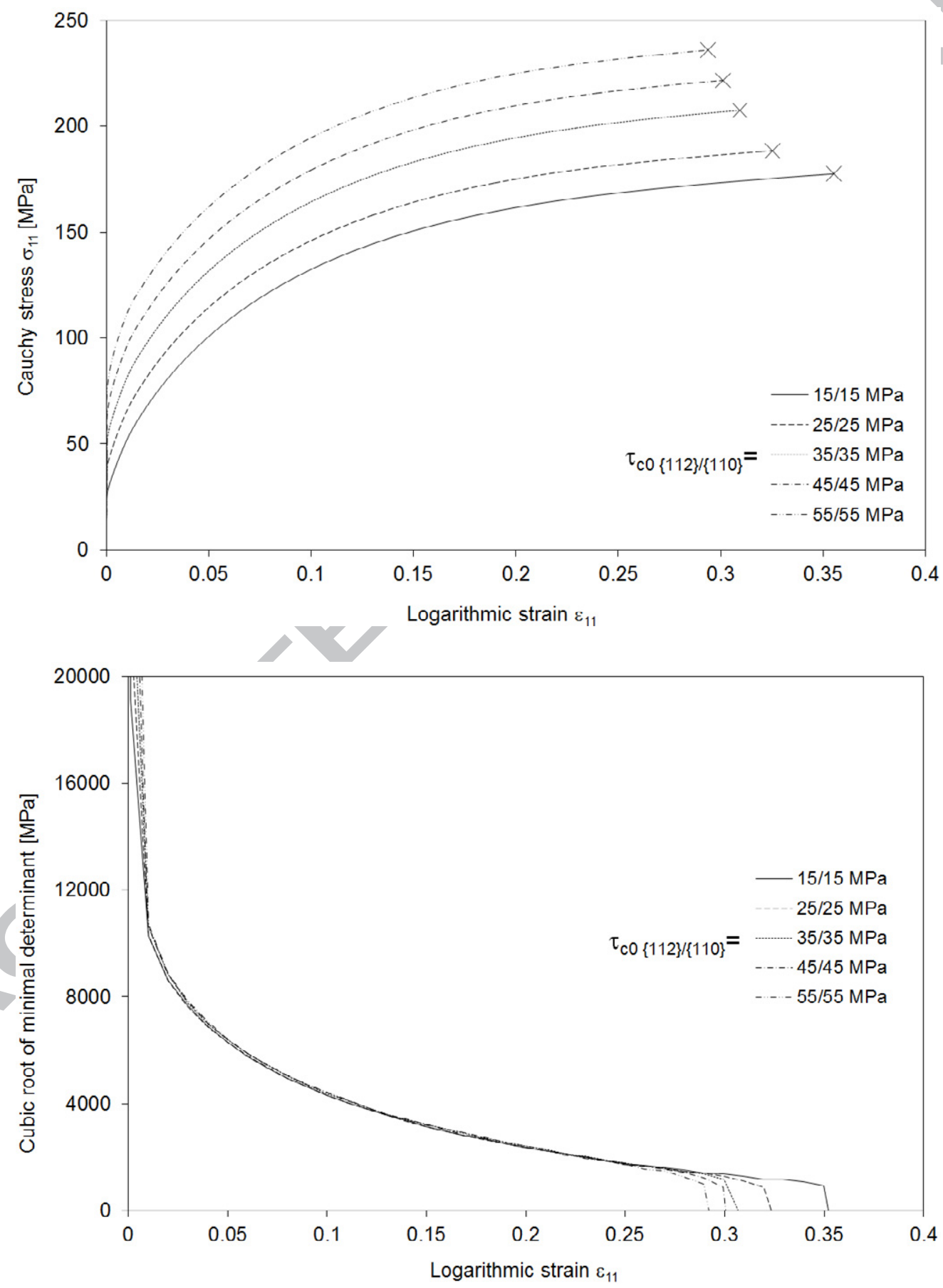
Fig. 13. Effect of the initial critical shear stress (of $\{110\}$ and $\{112\}$ slip plane families) on the ductility limit of a polycrystal (self-consistent scheme): Responses for plane strain tensile tests performed parallel to the rolling direction until the loss of strong ellipticity (top) and the minimal eigenvalue of the symmetric part of the acoustic tensor over all orientations of the normal to the localization band (bottom)
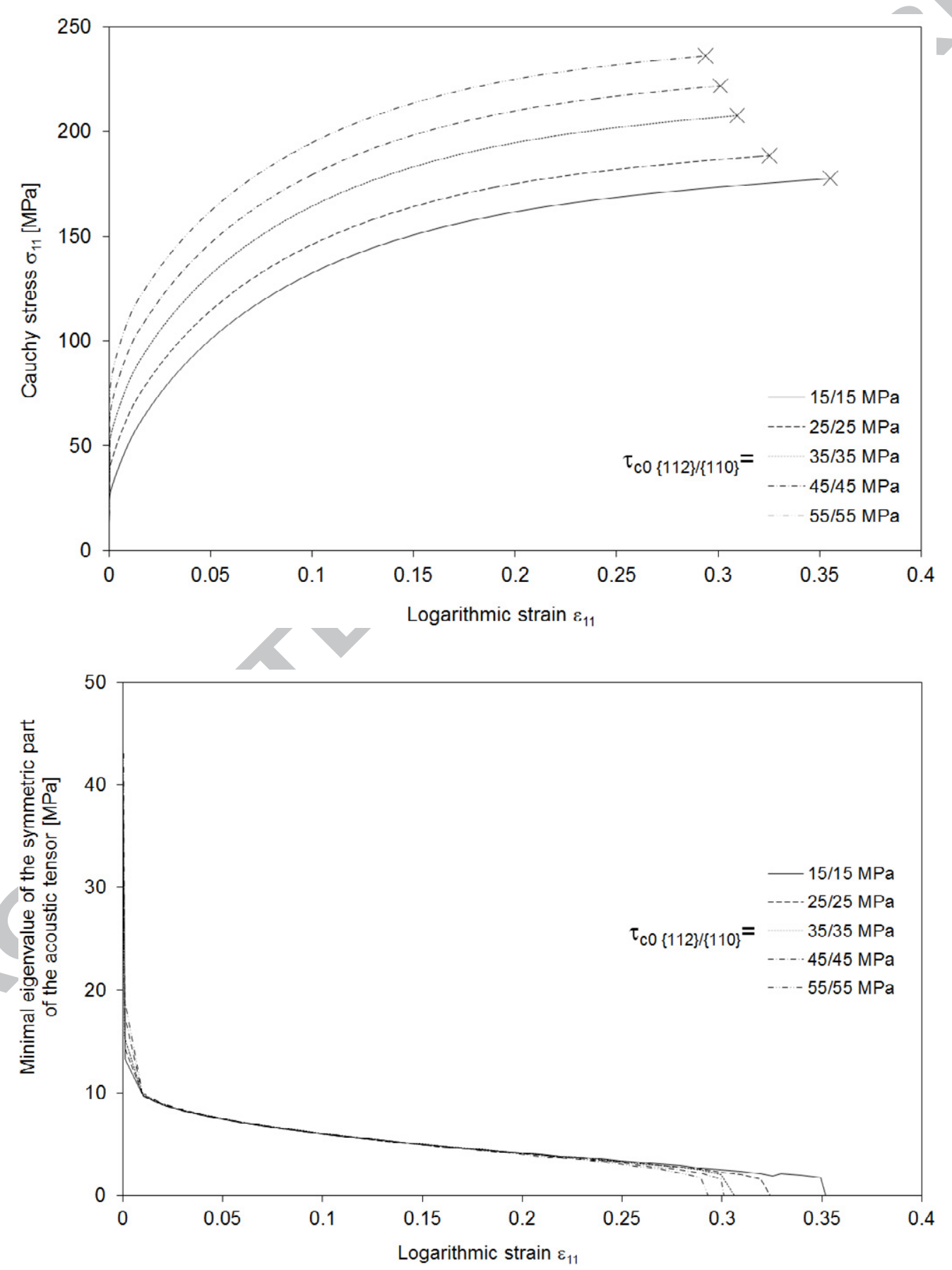
Fig. 14. Effect of the critical annihilation distance of dislocations on the ductility limit of a polycrystal (self-consistent scheme): Responses for plane strain tensile tests performed parallel to the rolling direction until the loss of ellipticity (top) and the minimal determinant of the acoustic tensor over all orientations of the normal to the localization band (bottom)
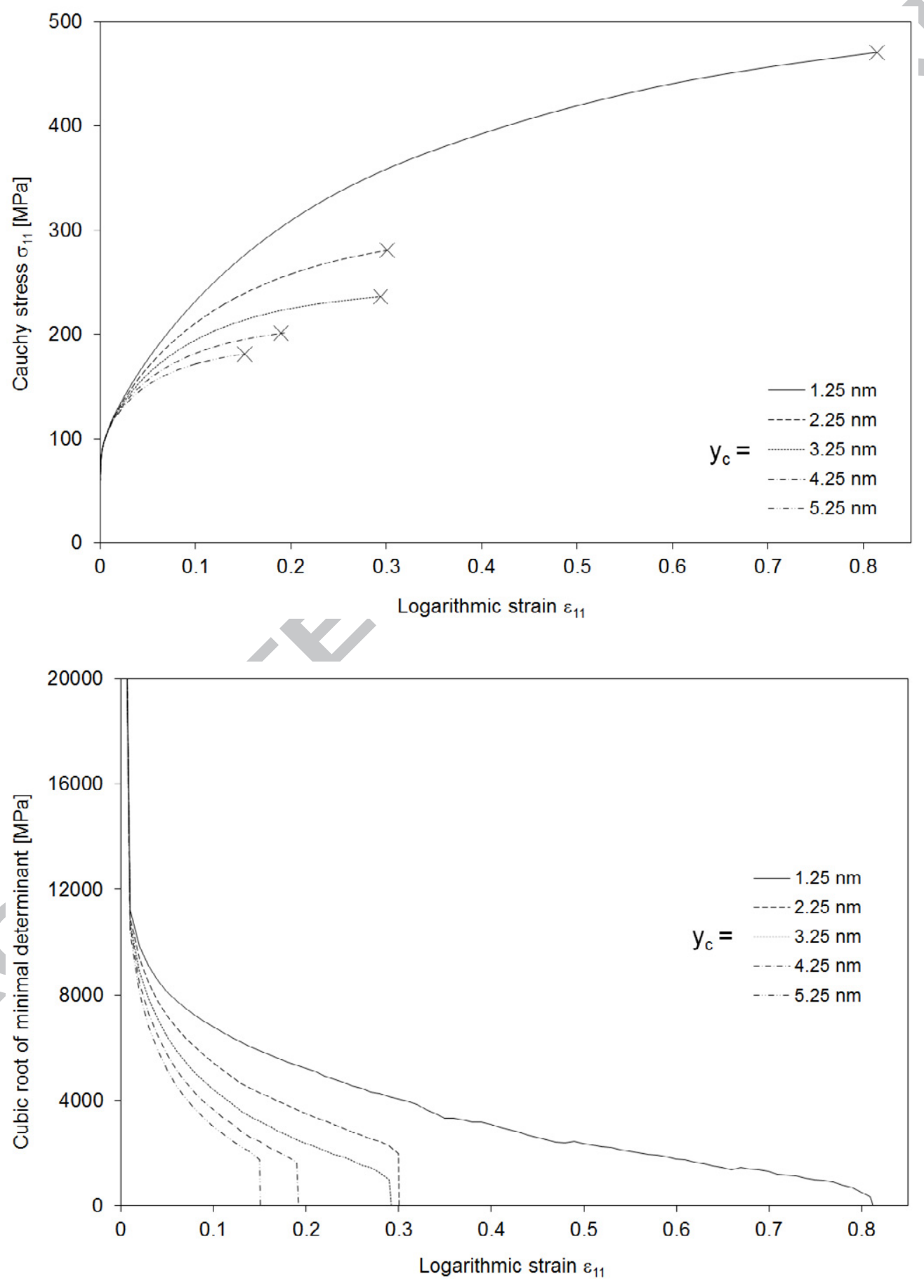
Fig. 15. Effect of the mean free path parameter on the ductility limit of a polycrystal (self-consistent scheme): Responses for plane strain tensile tests performed parallel to the rolling direction until the loss of ellipticity (top) and the minimal determinant of the acoustic tensor over all orientations of the normal to the localization band (bottom)
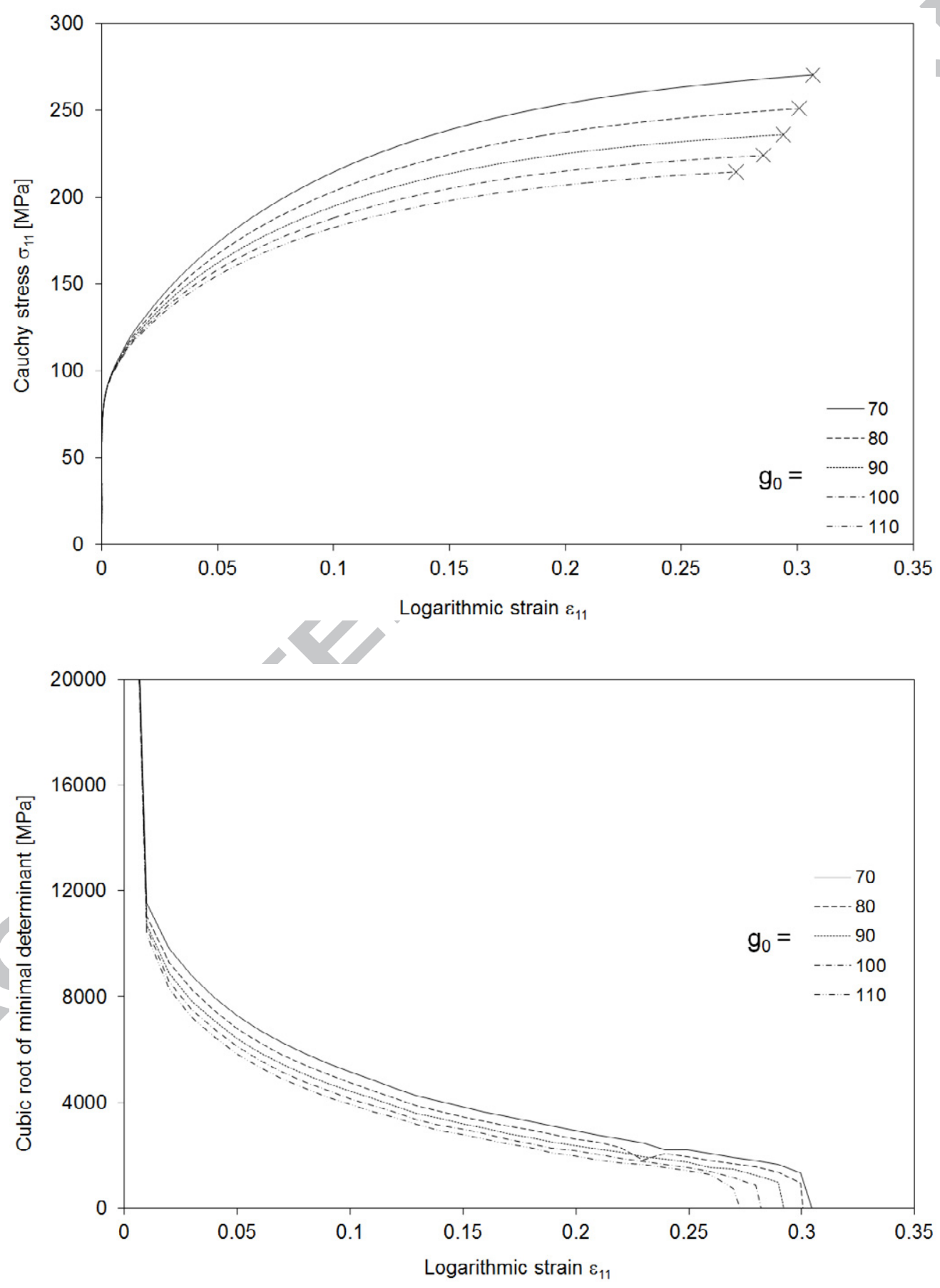
Fig. 16. Effect of the initial critical shear stress (of $\{110\}$ and $\{112\}$ slip plane families) on the ductility limit of a polycrystal (Taylor model): Responses for plane strain tensile tests performed parallel to the rolling direction until the loss of ellipticity (top) and until the loss of strong ellipticity (bottom)
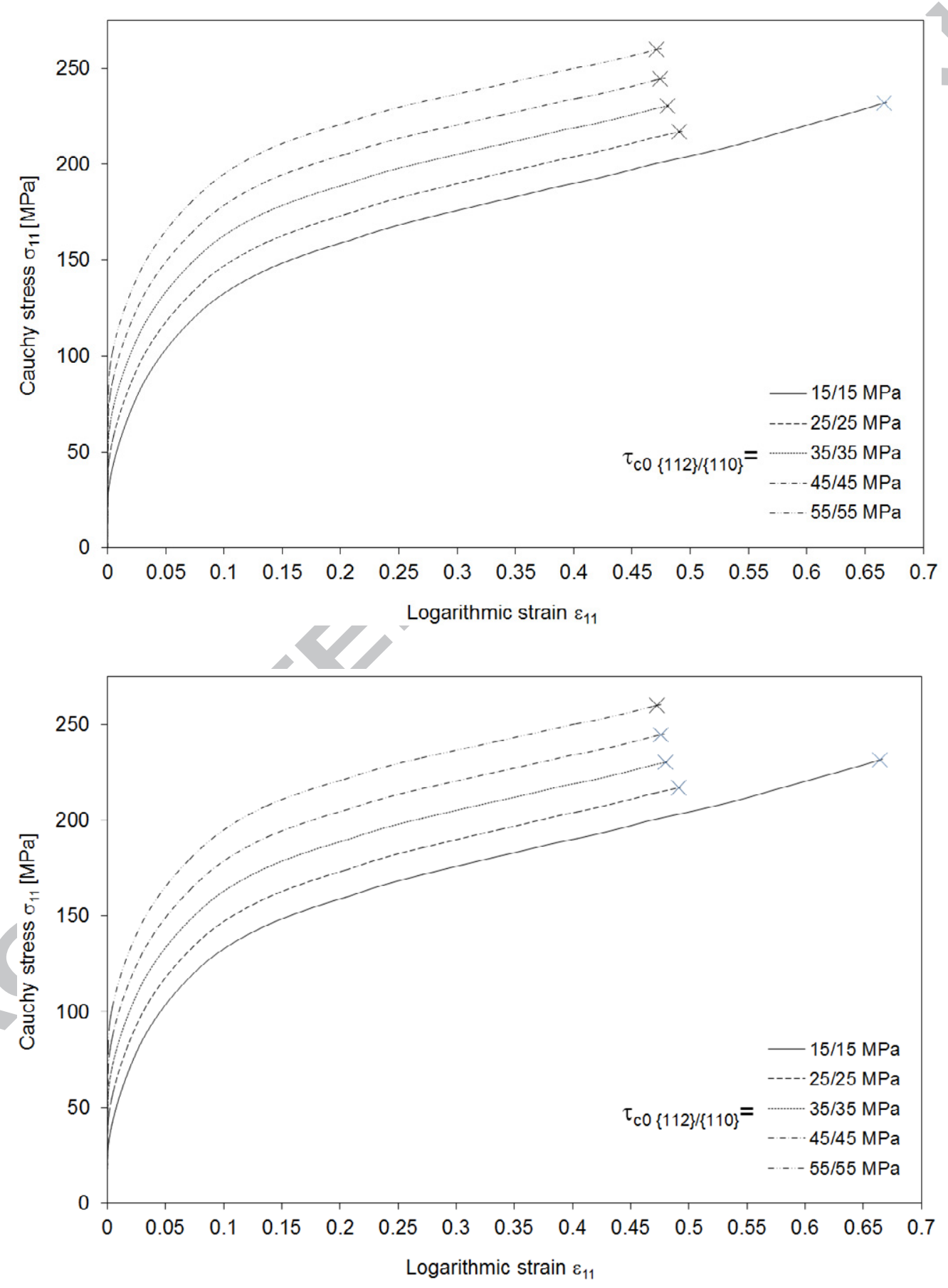
Fig. 17. Effect of the critical annihilation distance of dislocations (top) and of the mean free path parameter (bottom) on the ductility limit of a polycrystal (Taylor model):

Responses for plane strain tensile tests performed parallel to the rolling direction until the loss of ellipticity
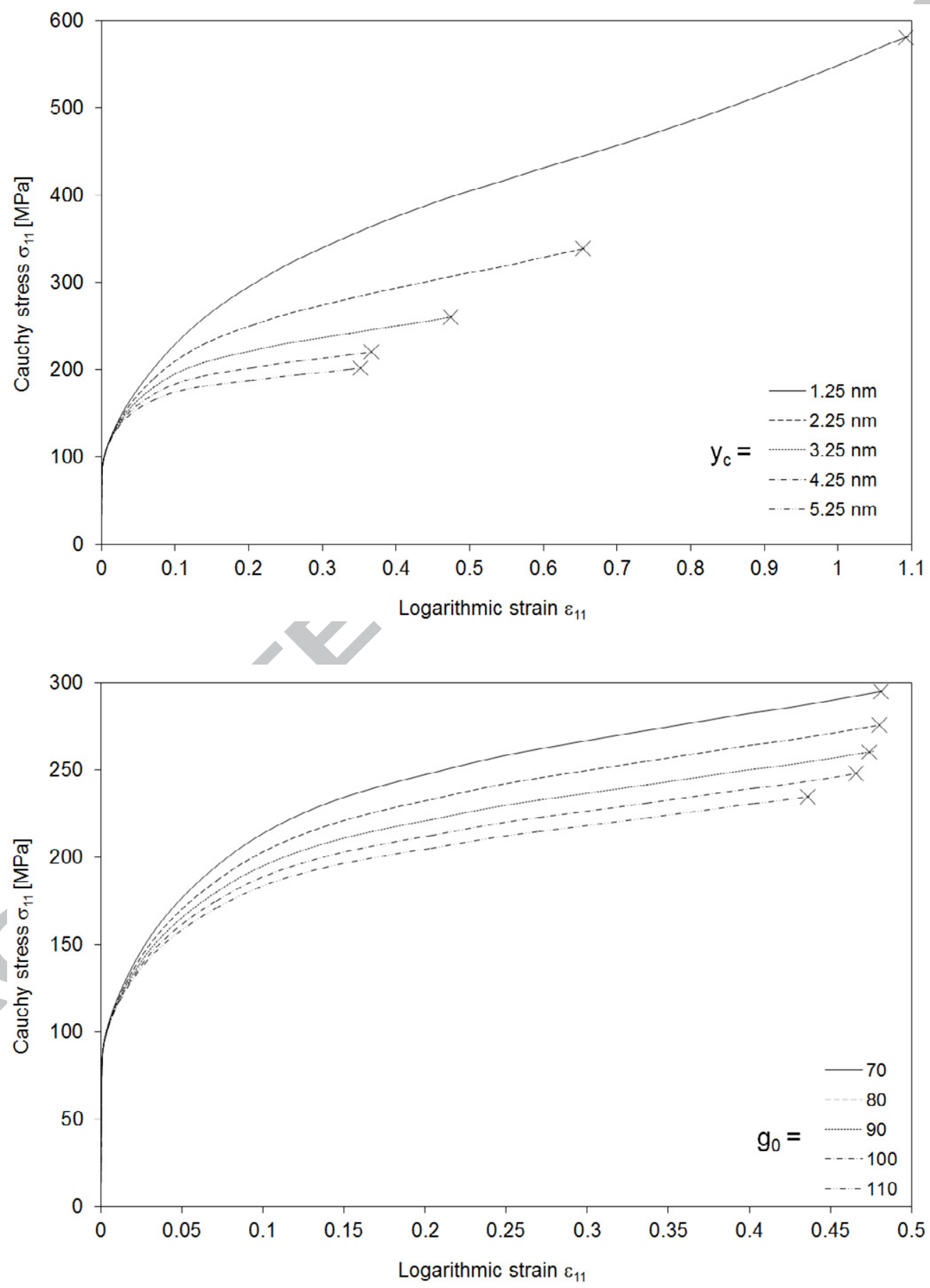
Fig. 18. Comparison between the proposed model and the experiments for the studied IF-Ti single-phase steel for different linear and sequential loading paths performed perpendicular to the rolling direction (PST 10\% SSh refers to a cross test consisting of 10\% Plane Strain Tension followed by Simple Shear, while BT refers to reverse shear tests at $10 \%, 20 \%$, and $30 \%$ of shear prestrain)

- Proposed model

$-\boxminus-$ Experiments

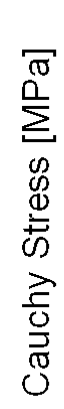

UT: Uniaxial Tension

एक SSh: Simple Shear

$\sum \quad$ BT: Bauschinger Test

PST $10 \%$ SSh: Orthogonal Test

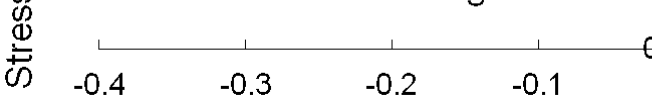

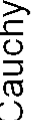

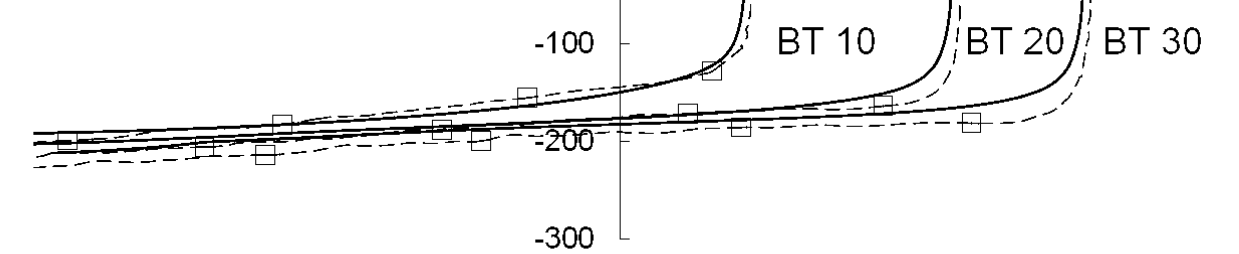

Strain / Amount of shear 
Fig. 19. FLDs associated with linear loading paths for the IF-Ti single-phase steel obtained with the proposed polycrystal self-consistent model (coupled with Rice's bifurcation criterion), and ArceloMittal's model

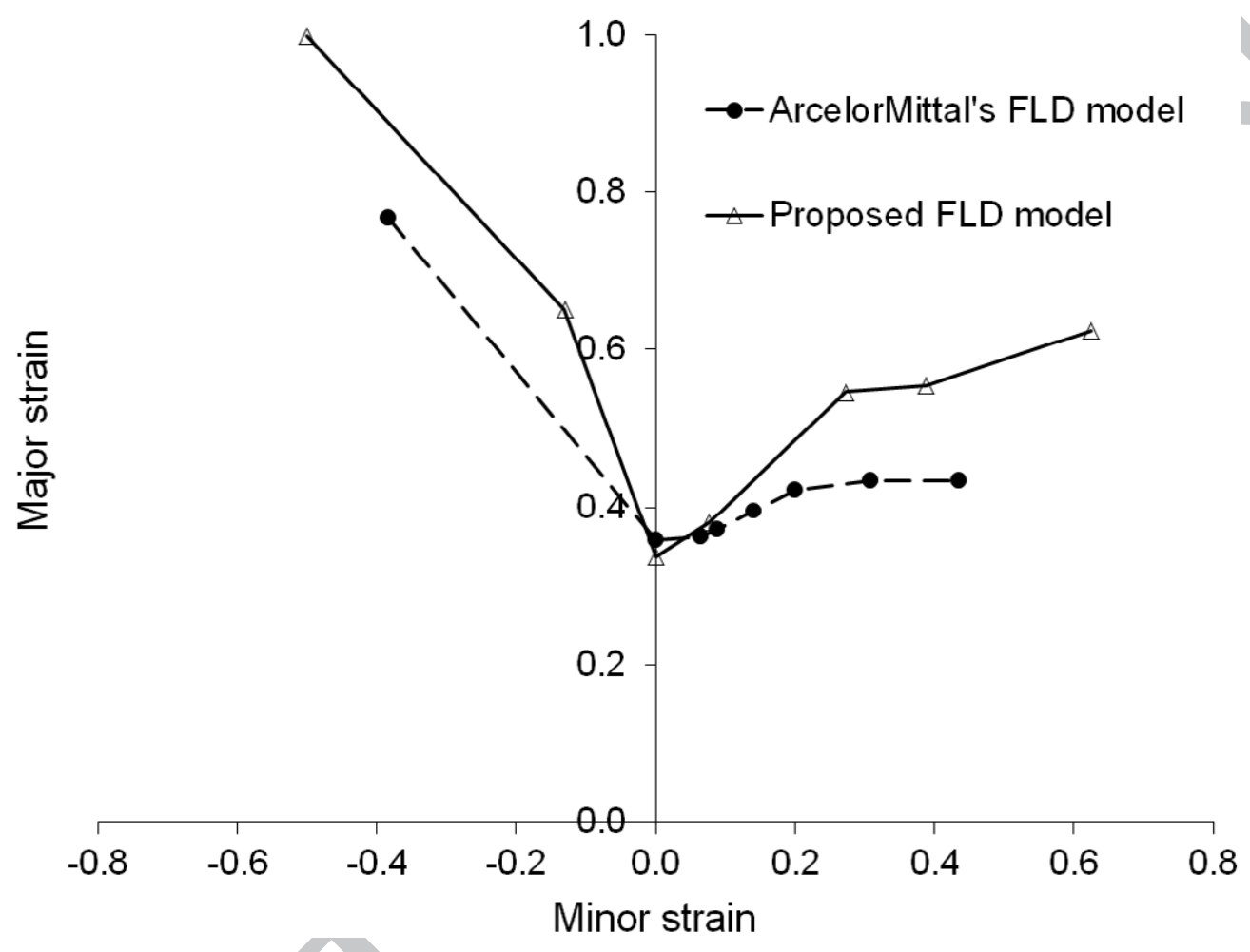


Fig. 20. FLDs associated with linear and sequential loading paths for the IF-Ti singlephase steel obtained with the proposed polycrystal self-consistent model coupled with Rice's bifurcation criterion: the sequential FLDs correspond to 5\% Uniaxial Tension (UT) prestrain and 5\% Balanced Biaxial Tension (BBT) prestrain, respectively

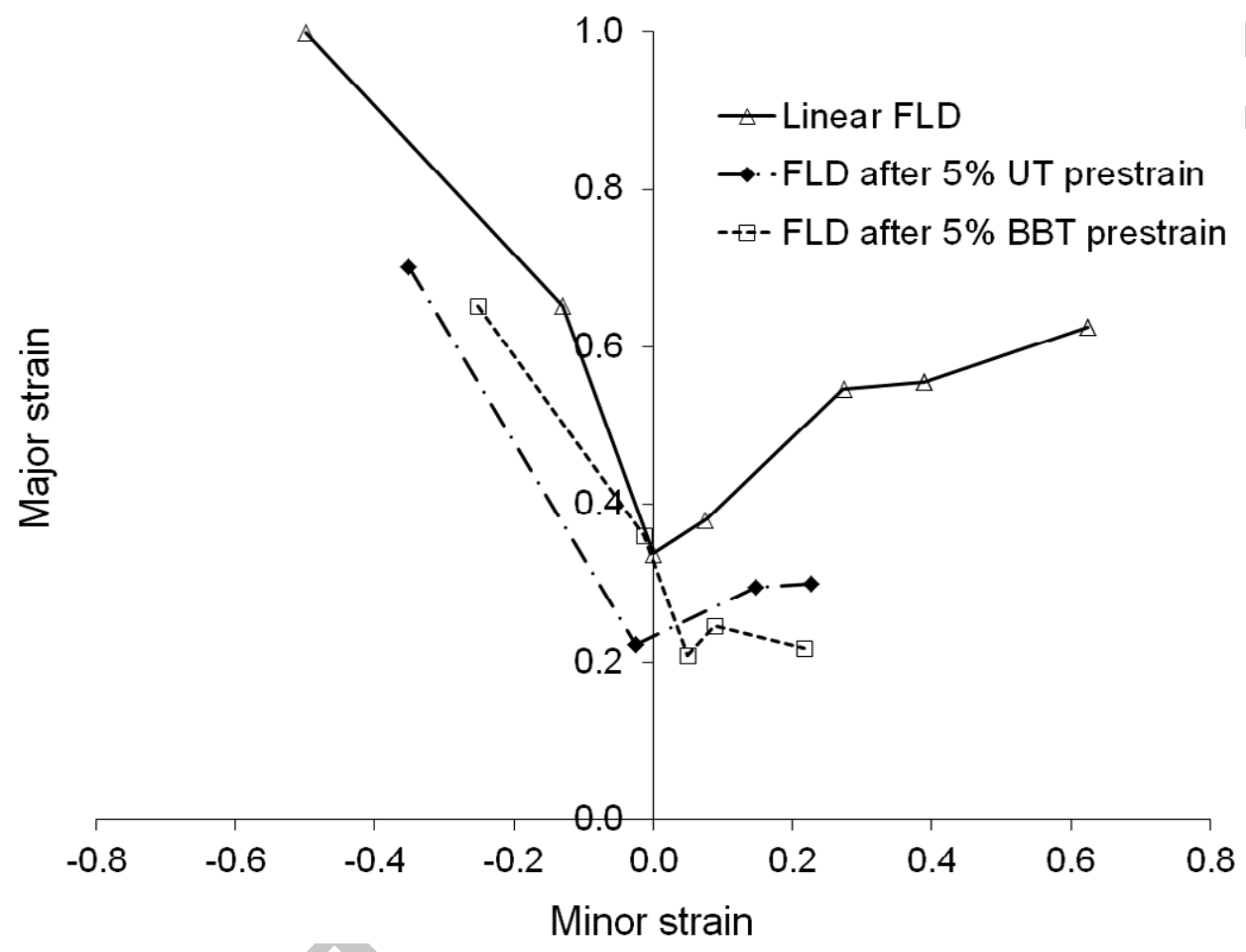




\section{Research highlights}

- Strain localization analysis is performed for single crystals and polycrystals.

- Relationships between microstructure-related parameters and ductility are disclosed.

- Large-strain elastic-plastic modeling based on self-consistent scale transition is used.

- FLDs are constructed based on bifurcation theory and loss of strong ellipticity.

- Results of the self-consistent scheme and the full-constraint Taylor model are compared. 